Dirk Naujoks

\title{
Ion Confinement in a Linear
}

\section{Plasma Column}

IPP $8 / 18$

November 2001 


\section{MAX - PLANCK - INSTITUT FÜR PLASMAPHYSIK}

GARCHING BEI MÜNCHEN

Ion Confinement in a Linear Plasma Column

Dirk Naujoks

IPP $8 / 18$

November 2001

Max-Planck-Institut für Plasmaphysik, EURATOM Association Bereich Plasmadiagnostik, Mohrenstr. 41, 10117 Berlin ved." 


\title{
ION CONFINEMENT IN A LINEAR PLASMA COLUMN
}

\author{
D. Naujoks \\ Max-Planck-Institut für Plasmaphysik, EURATOM Association, \\ Bereich Plasmadiagnostik, \\ Mohrenstr. 41, D-10117 Berlin, Germany
}

\begin{abstract}
The behavior of magnetized plasmas concerning charge shielding, polarization and ion confinement has been investigated by means of a PIC (particle-in-cell) model. It is shown that the dimensionless ratio $\chi=n_{p l} m /\left(\epsilon_{o} B^{2}\right)$ being the dielectric susceptibility (with the plasma density $n_{p l}$, the mass $m$ and the magnetic field strength $B$ ) is the main parameter in the analysis. For small values of $\chi$ the plasma is not able to compensate additional test charges and shielding does not occur. For high values the effect of the magnetic field vanishes and the electric forces dominate.

Under the conditions of today's fusion experiments the electrons are usually tied to the magnetic field lines due to their small gyration radii $\rho_{e}$ which are comparable to the Debye length $\lambda_{D}$. The ions, on the other hand, have much larger gyration radii $\rho_{i} \gg \lambda_{D}$ and their ratio $\chi_{i}$ exceeds 1000 . It is shown that for such high values the effect of the magnetic field is so weak, that only electric forces are of importance for the ion confinement in a plasma column.
\end{abstract}

\section{Introduction}

The behavior of magnetized plasmas is quite complex and often not accessible by analytical models. Whereas the problem of charge shielding in plasmas is described in detail in every text-book on plasma physics, the same process of charge shielding does not allow such a simple analytical treatment in the case of magnetized plasmas.

Hence, numerical modeling is required. For this purpose the PIC (particle-in-cell) code PLAS was developed (Sec. 2) with the aim to model a plasma containing two species of opposite charge state embedded in an external magnetic field. Several simulations have 
been performed for a wide range of plasma parameters. It turned out that the ratio of the gyro radius $\rho$ to the Debye length $\lambda_{D}$ is the main parameter

$$
\xi_{e, i}=\frac{\rho_{e, i}}{\lambda_{D}}=\sqrt{\frac{n_{p l} m_{e, i}}{\epsilon_{o} B^{2}}}=\left(\chi_{e, i}\right)^{2}
$$

where $m_{e}$ and $m_{i}$ denote the masses of species 'e' and 'i', respectively, and $\epsilon_{o}$ is the permittivity of free space. Note, that this ratio does not depend on the temperatures. In the derivation of (1) the relation $m\left\langle v^{2}\right\rangle / 2=\mathrm{k} T / 2$ was used.

For example, the parameter $\xi$ determines the shielding behaviour of a magnetized plasma (Sec. A). In the case of a strong magnetic field and small mass (i.e. small value of $\xi$ ) the charged particles are fixed to the magnetic field lines so that a shielding across $B$ is not possible (it is, of course, still possible along $B$ ). If the gyration radius is much larger than the Debye length (i.e. $\xi \geq 30$ ) then the shielding is almost perfect as in the magnetic-field-free case [1].

The second part of the paper deals with the confinement of electrons and ions in a plasma column with an axial magnetic field. In the PLAS calculations the temporal evolution of the plasma is calculated starting with a given density profile and with isotropic velocity distributions, thus simulating a switch-on of a magnetic field at time $t=0$. No external heating or cooling is considered. It is shown (Sec. 3) that the difference of the electron and ion gyration radii leads to charge separation resulting in the origin of an electric field. Under the usual conditions of plasma and fusion experiments the electrons are confined by the magnetic field, whereas the plasma ions (as fluid) are held entirely by the self-generated electric field. Whereas the electrons with their extremely small gyration radii are relatively fixed at the magnetic field lines, the ions need this additional force which ensures the nearly equality of the electron and ion density profiles to the required high degree.

In fact, if a small plasma region with an extent of several Debye length is considered the electrons are trapped in this small region after the magnetic field is switch on, since their gyration radii are of the same order as the Debye length. However, the heavier ions with their much larger gyro radii will leave this region after a short time, i.e. charge separation occurs leading to electric field generation. Without density gradients adjoining plasma cells are able to provide each other with the required in- and out-going ion fluxes in order to sustain overall charge balance, but this is not possible if there is a density (or temperature) gradient. In this case, a confining electric field is required and is inevitable.

\section{Model description}

In the simulations two species of particles are used: negative charged particles with mass $m_{e}$ and positive charged particles with mass $m_{i}$. The subscripts $e$ and $i$ are taken in the 


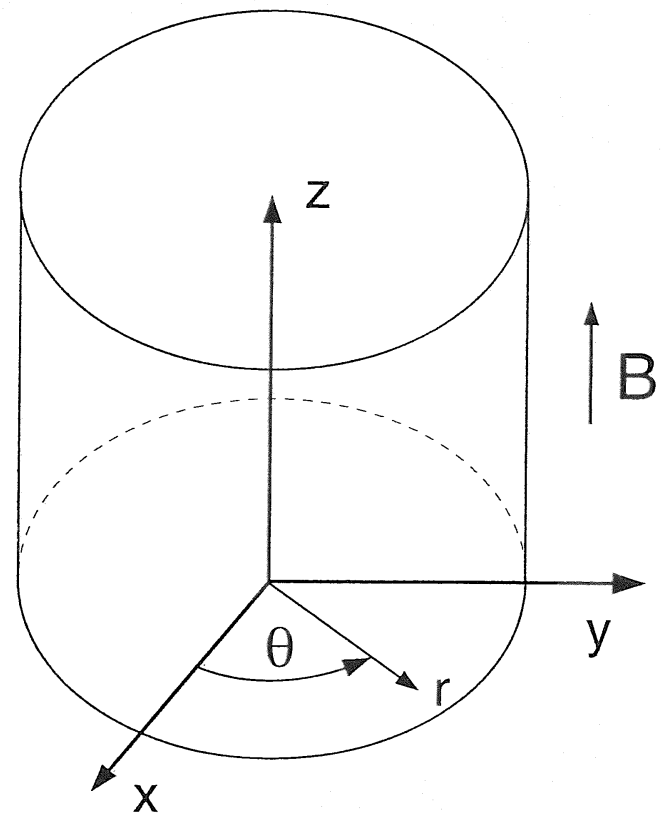

Figure 1: model geometry

following also for the denotation of other physical parameters such as temperature and density.

A large number of particles $N^{\max }$ (up to several $10^{5}$ particles for each species) is started uniformly distributed on the plane $(x, y)$ (see Fig.1) with a Maxwellian velocity distribution generated using the method of Box and Muller [2] which results in the following initial velocity components

$$
\begin{aligned}
& v_{x o}=\sqrt{\mathrm{k} T_{e, i} / m_{e, i}} \sqrt{-2 \ln R_{1}} \cos \left(2 \pi R_{2}\right) \\
& v_{y o}=\sqrt{\mathrm{k} T_{e, i} / m_{e, i}} \sqrt{-2 \ln R_{1}} \sin \left(2 \pi R_{2}\right) \\
& v_{z o}=0
\end{aligned}
$$

$R_{1}$ and $R_{2}$ are two random numbers, which are uniformly distributed over the range $[0,1]$. The dynamics of the particles is determined by the fixed external magnetic field $\boldsymbol{B}=$ $(0,0, B)$ (Fig.1) and the internal electric field $\boldsymbol{E}$. Cylindrical symmetry is assumed, so that $\partial / \partial y=\partial / \partial z=0$.

The motion of each single particle (i.e. for both $e$ and $i$ particles) is calculated by means of the equations

$$
m \frac{d \boldsymbol{v}}{d t}=q[\boldsymbol{E}+\boldsymbol{v} \times \boldsymbol{B}], \quad \boldsymbol{v}=\frac{d \boldsymbol{r}}{d t},(\boldsymbol{r}=x \hat{\boldsymbol{x}}+y \hat{\boldsymbol{y}})
$$

Both species are assumed to be singly charged $\left(q_{e}=-e, q_{i}=e\right)$. Eq.(3) is solved using the 'leap-frog'-method for the integration and the $[\boldsymbol{v} \times \boldsymbol{B}]$ rotation has been implemented following Boris (see in [3] or in [2]). 
Knowing the spatial positions of the particles $r=(x, y)$ at a certain time the temporary density profile of both species $n_{e, i}$ is deduced using the general neutrality condition $\int n_{i}(r) d V=\int n_{e}(r) d V=n_{p l} V$ leading to

$$
n_{e, i}(r, t)=\frac{n_{p l} V_{p l}}{N^{\max }} \frac{\Delta N_{e, i}(r, t)}{\Delta V}
$$

where $\Delta N_{e, i}$ is the number of particles in the volume element $\Delta V=2 \pi r \Delta z \Delta r$ at the position $r$ and at the time $t$. $V_{p l}$ is the plasma volume, $\Delta r$ is the width of the cells in the $r$-direction. The factor $\left(n_{p l} V_{p l} / N^{\max }\right)$ in (4) takes into account, that in the simulations only $N^{\max }$ so called 'representive' particles are used instead of the real number of particles $n_{p l} V_{p l}$ of the considered plasma. The plasma extension $\Delta z$ in the $z$ direction has no effect in the present calculations $(\Delta z=1 \mathrm{~m}$ is taken in all calculations where it was necessary to ensure the right units). Finally, $n_{p l}$ denotes the plasma density. In the code the point charges are assigned to its nearest grid point by linear interpolation (particle-incell method).

The electric field $E_{r}$ (one-dimensional in radial direction) is determined by the charge density distribution using the Poisson equation

$$
\begin{aligned}
\nabla^{2} \phi & =\frac{1}{r} \frac{\partial \phi}{\partial r}+\frac{\partial^{2} \phi}{\partial r^{2}}=\frac{e}{\epsilon_{o}}\left[n_{e}(r)-n_{i}(r)\right] \\
E_{r} & =-\frac{\partial \phi}{\partial r}
\end{aligned}
$$

(where $\epsilon_{o}$ is the permittivity of free space). The application of finite differences on a uniform mesh leads to a tridiagonal set of equations, which is readily solved using Thomas algorithm (see in [4]) with forward elimination and backward substitution. The electric field force is also linearly interpolated for positions between two grid points, exactly as in the charge assignment. In the calculation the cell size $\Delta r$ is kept well below the Debye length and the time step $\Delta t$ in the particle moving calculations is chosen to satisfy the Courant-Friedrichs-Lewy criterion $1>\left(v_{e, i}^{\max } \Delta t\right) / \Delta r>0.1$ [5].

The whole set of particles is followed in time by calculating Eqs. $(3,4,6,5)$ with the boundary conditions for the potential

$$
\left.\phi\right|_{r=R}=0 \quad \text { and }\left.\quad \frac{\partial \phi}{\partial r}\right|_{r=0}=0
$$

where $R$ is the radius of the vessel wall.

\section{Linear plasma column}

The PLAS code (Sec. 2) has been used to model a linear plasma column radially confined in a magnetic field $B=\left(0,0, B_{z}\right)$ with the plasma radius $r_{p l}$. Contact of the plasma 
column with the wall has been not allowed by choosing a sufficient large radius of the wall $R$, i.e. $R>r_{p l}+2 \rho$.

In the analysis three cases are distinguished: (i) both species have the same gyration radius, $\rho_{i}=\rho_{e}$ (Sec. 3.2), (ii) their gyration radii are different, $\rho_{i}>\rho_{e}$ (Sec. 3.3), and (iii) the parameter $\xi_{i}$ is much larger than $\xi_{e}, \xi_{i} \gg \xi_{e}$ (Sec. 3.4).

The following idealizations are applied: (i) cross field diffusion due to collective transport effects such a plasma turbulence is not considered, since the resulting change of the density profiles in time would be much slower than the occurence of charge separation at the plasma edge, (ii) the ion motion is considered only in the $(x, y)$-plane perpendicular to the axial magnetic field, (iii) the electric field and potential are calculated only for the radial direction according to Eqs.(6,5), (iv) effects caused by endplates are not considered.

\subsection{Initial density profiles}

In the simulations particles are started with a certain density profile $n_{e, i}(r)$, which are generated using random numbers. Generally, the radial profile $n(r)$ is related to random numbers $R_{k}$, which are uniformly distributed in the interval from 0 to 1 , by

$$
R_{k}=\frac{\int_{0}^{r_{k}} n(r) r d r}{\int_{0}^{R} n(r) r d r}
$$

For given $R_{k}$ ( $k$ runs from 1 to $N^{\max }$ ) Eq.(8) is to be solved in order to obtain the starting positions $r_{k}$. For example, an analytical solution is possible in the case of a step-profile

$$
n(r)=\left\{\begin{array}{rll}
n_{p l} & \text { for } & r \leq r_{p l} \\
0 & \text { for } & r>r_{p l}
\end{array}\right.
$$

Substituting for $n(r)$ from (9) the integration of (8) gives $R_{k}=r_{k}^{2} / r_{p l}^{2}$ which leads to the well-known relation $r_{k}=\sqrt{R_{k}} r_{p l}$, generating a uniform density in the border of $r_{p l}$. More realistic profiles can be represented as

$$
n(r, t)=\sum_{i=1}^{\infty} \alpha_{i} J_{o}\left(\frac{\mu_{i}}{R} r\right) \exp \left[-\left(\frac{\mu_{i}}{R}\right)^{2} D_{\perp} t\right]
$$

where $\mu_{i}$ are the zeros of the Bessel function $J_{o}$ (for $\left.i \geq 30 \mu_{i} \simeq \pi(i-1 / 4)\right) ; r_{p l}$ is the plasma radius and $R$ is the radius of the chamber. Eq.(10) is the solution of the continuity equation in cylindrical geometry [6]

$$
\frac{1}{D_{\perp}} \frac{\partial n}{\partial t}=\frac{\partial^{2} n}{\partial r^{2}}+\frac{1}{r} \frac{\partial n}{\partial r}
$$


where the diffusion coefficient is taken constant $D_{\perp}=$ const. and the boundary condition $n(r=R, t)=0$ is applied. If the starting profile $n(r, t=0)$ is equal to $n_{p l}$ for $r \leq r_{p l}$ and elsewhere zero (i.e. given by (9)), the coefficients $\alpha_{i}$ are [6]

$$
\alpha_{i}=\frac{2}{R^{2}\left[J_{1}\left(\mu_{i}\right)\right]^{2}} \int_{0}^{R} n_{e}(r, 0) J_{o}\left(\frac{\mu_{i} r}{R}\right) r d r=\frac{2 n_{p l} r_{p l}}{\mu_{i} R} \frac{J_{1}\left(\mu_{i} r_{p l} / R\right)}{\left[J_{1}\left(\mu_{i}\right)\right]^{2}}
$$

Density profiles calculated for three values of the diffusion parameter $d=\sqrt{D_{\perp} t}=1,3,10$ $m m$ are shown in Fig.2. The use of such density profiles allows to investigate the effect of

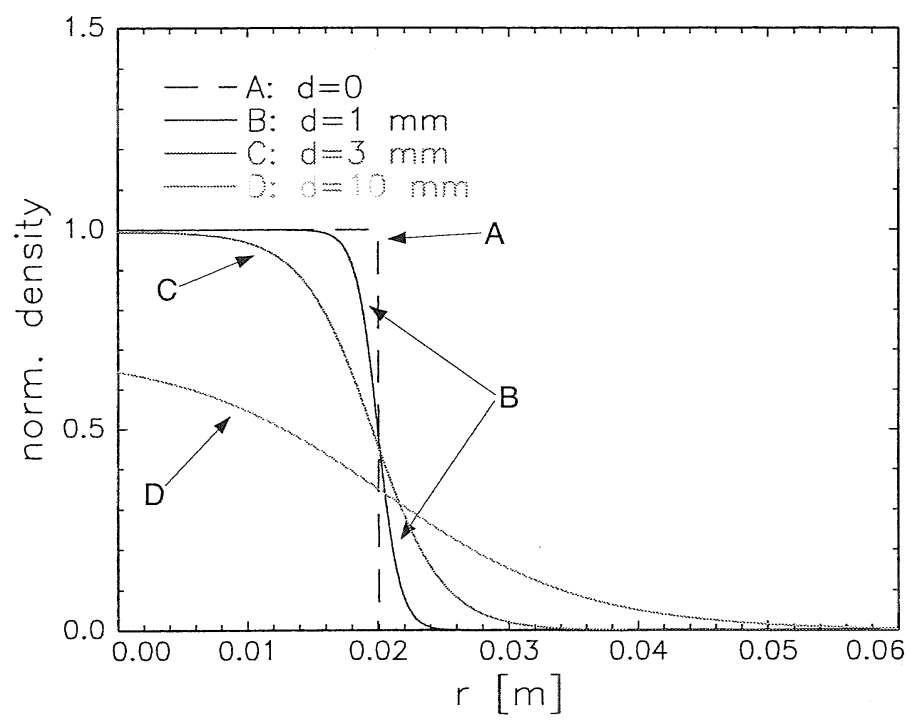

Figure 2: Normalized density profiles presented as Fourier-Bessel series for different $d=\sqrt{D_{\perp} t}$ as indicated in the figure.

a density gradient on the process of charge separation at the plasma edge. As mentioned before, the diffusion process itself is here not the matter of consideration, i.e. these density profiles serve only as a more or less realistic starting condition for the simulations of the charge separation effect, which is expected to dominate the plasma behavior on a much shorter timescale than cross-field diffusion does.

\subsection{Case $\rho_{i}=\rho_{e}$}

As initial density profile (at $t=0$ ) a distribution of type $C$ (Fig.2, with $d=3 \mathrm{~mm}$ ) has been assumed for both species $e$ and $i$. The motion of the particles is then followed in time. Since equal gyration radii are taken $\left(\rho_{i}=\rho_{e}\right)$ the resulting density profiles (averaged over some gyration times) are equal, $n_{i}(r)=n_{e}(r)$, but due to the finite gyration radius they are broader in comparison to the starting profile. Charge separation does not occur and the electric field is zero. The results of the simulations are shown in Fig.3. As expected, the mean rotation velocities of both species $v_{\theta}^{e, i}$ are obtained to be equal to their respective 'diamagnetic drift' velocity

$$
v_{\text {diam }}=\frac{B \times \nabla p}{n q B^{2}}
$$



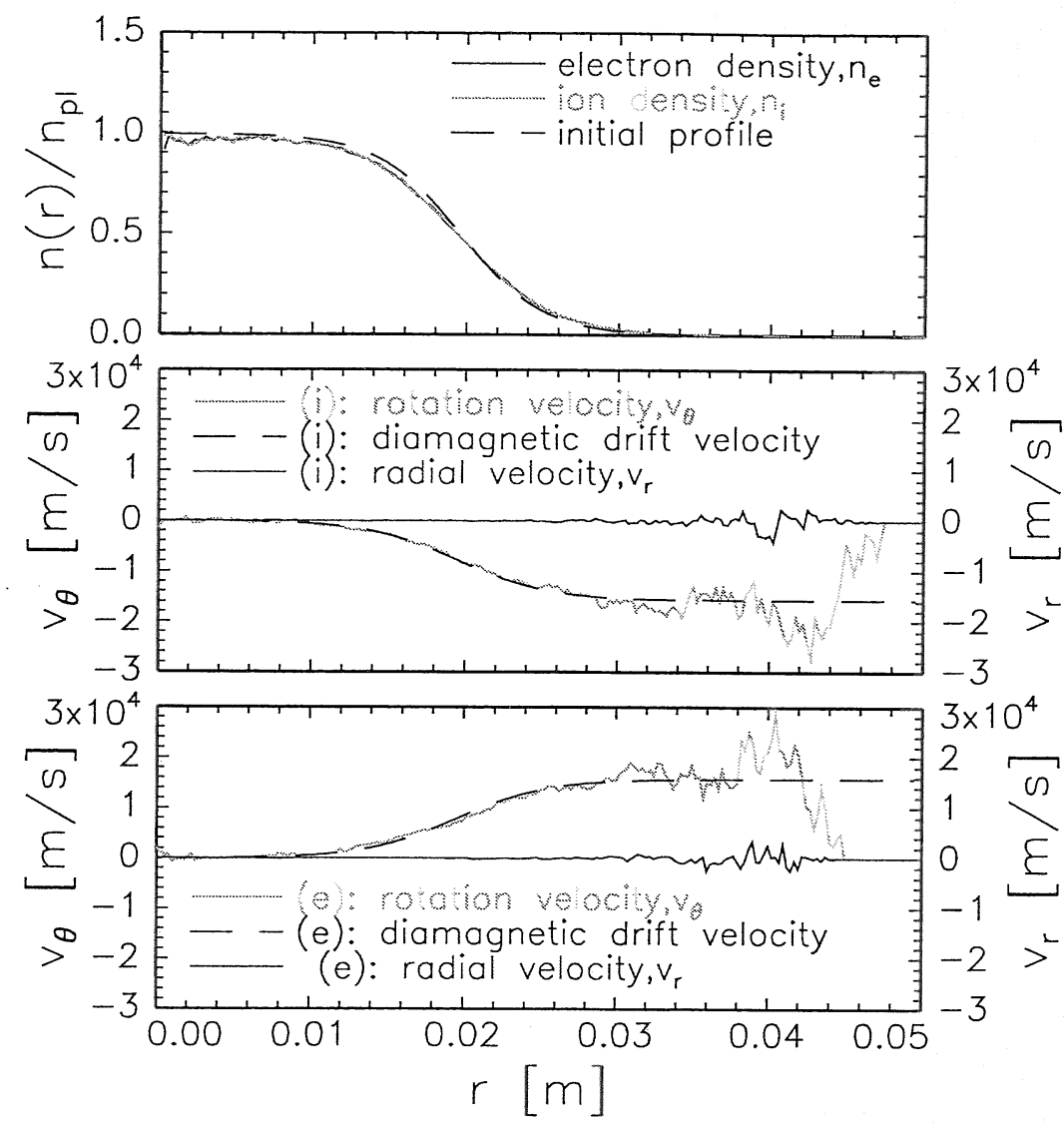

Figure 3: Mean radial profiles of $n$, $v_{r}$ and $v_{\theta}$ for both species $e$ and $i$. (parameters: $R=$ $0.05 m, n_{p l}=10^{15}$ $m^{-3}, B=0.22 T$, $T_{e}=T_{i}=10 \mathrm{eV}$, $m_{i}=m_{e}=1 \mathrm{amu}$, $\xi_{i}=\xi_{e}=2, \rho_{i}=$ $\rho_{e}=1.5 \mathrm{~mm}$ )

which gives in cylindrical geometry for constant temperatures

$$
v_{d i a m}^{i}(r)=\frac{\mathrm{k} T_{i}}{e n_{i}(r) B_{z}} \frac{\partial n_{i}(r)}{\partial r}, \quad v_{d i a m}^{e}(r)=-\frac{\mathrm{k} T_{e}}{e n_{e}(r) B_{z}} \frac{\partial n_{e}(r)}{\partial r} .
$$

The values of $v_{\text {diam }}^{e, i}$ according to Eq.(14) are shown in Fig.3 by dashed lines. The origin of this drift is illustrated in Fig. 4 and can be understood by simply adding the gyrations orbits in the presence of a density or temperature gradient. This drift is a 'fluid' drift in the sense that the diamagnetic drift velocity represents the azimuthal velocity of all particles $\bar{v}_{\theta}(r)$ averaged in a certain volume element near $r$.

With the resulting drift around the main axis the particles have a total angular momentum

$$
L_{e, i}=m_{e, i} \int_{V} n_{e, i}(\boldsymbol{r} \times \boldsymbol{v}) d V=2 \pi \Delta z m_{e, i} \int_{0}^{R} n_{e, i} \bar{v}_{\theta}^{e, i} r^{2} d r
$$

Replacing the mean azimuthal velocity $\bar{v}_{\theta}^{e, i}$ by $v_{\text {diam }}^{e, i}$ (Eq.(13)) yields

$$
L_{e, i}^{\operatorname{diam}}=-\frac{4 \pi m_{e, i} \Delta z k T_{e, i}}{q_{e, i} B_{z}} \int_{0}^{R} n_{e, i} r d r=-\frac{2 k T_{e, i}}{\omega_{c}^{e, i}} N
$$




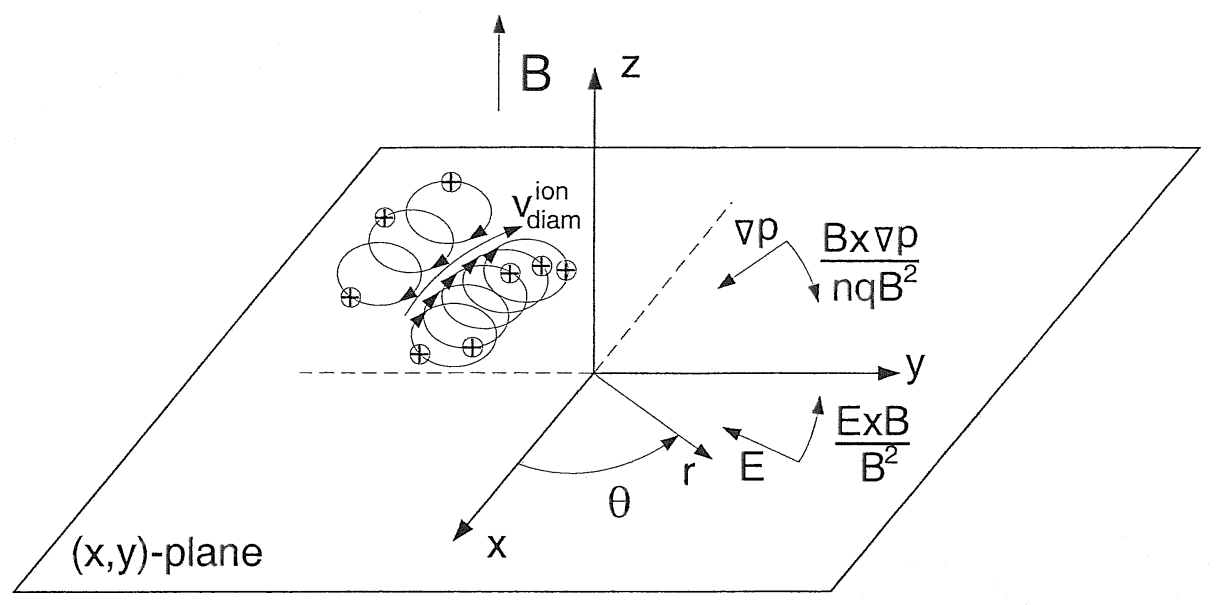

Figure 4: Model geometry with diamagnetic and $(\boldsymbol{E} \times \boldsymbol{B})$-drifts.

since $\int_{0}^{R}(\partial n / \partial r) r^{2} d r=-2 \int_{0}^{R} n r d r . \omega_{c}$ is the gyro frequency and $N$ is the total number of particles in the considered plasma volume $\left(N=N_{e}=N_{i}\right)$. $L$ is positive for species $e$ $\left(q_{e}=-e\right)$, and negative for species $i\left(q_{i}=+e\right)$. The oscillating behavior of the angular momentum and the radial currents are shown in Fig. 5 . It is caused by the periodic return of all particles to their initial positions after each gyration time.

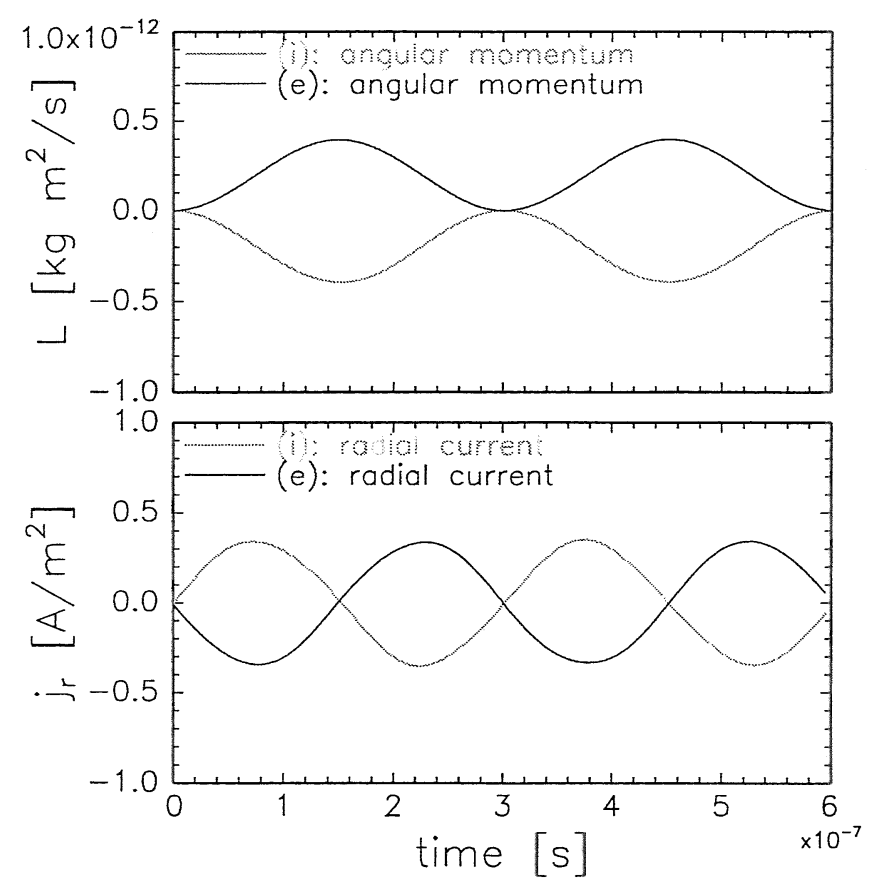

Figure 5: Temporal behavior of $L_{e, i}$ and $j_{r}^{e, i}=$ $q n_{e, i} v_{r}^{e, i}$. The latter value has been averaged over the plasma volume for each time step. (parameters as given in Fig.3, gyration time $\tau_{\rho, i}=\tau_{\rho, e}=3 \times 10^{-7} \mathrm{~s}$, $L_{e}^{\text {diam }}=-L_{i}^{\text {diam }}=2 \times 10^{-13}$ $\left.k g \mathrm{~m}^{2} / \mathrm{s}\right)$

Despite the fact that the mean radial velocities $\left(v_{r}^{e, i}\right)$ and currents $\left(j_{r}^{e, i}\right)$ equal zero (Fig.3 and Fig.5) the mean angular momentums of both species are nonzero and are given by relation (16). This gain of angular momentum, which was zero at the start of the 
simulation, is provided by the magnetic field force

$$
\frac{d L_{e, i}}{d t}=\int_{V}(\boldsymbol{r} \times \boldsymbol{F}) d V=\int_{V} \boldsymbol{r} \times\left(\boldsymbol{j}_{e, i} \times \boldsymbol{B}\right) d V=-2 \pi \Delta z B_{z} q_{e, i} \int_{0}^{R} n_{e, i} v_{r}^{e, i} r^{2} d r
$$

The radial currents flowing during half the gyration time (Fig.5) can be estimated as $j_{r}^{i} \approx\left(k T_{e, i} n_{e, i}\right) /\left(B_{z} r_{p l}\right)\left(j_{r}^{e}=-j_{r}^{i}\right)$ where the value for the density should be taken at position $r_{p l}$ (in this analysis $r_{p l} \approx 0.02 \mathrm{~m}$, Fig.3). Note, that the total radial current $j_{r}=j_{r}^{i}+j_{r}^{e}$ is zero at all time moments, and subsequently the corresponding torque $\int \boldsymbol{r} \times(\boldsymbol{j} \times \boldsymbol{B}) d V$ also equals zero.

\subsection{Case $\rho_{i}>\rho_{e}$}

The results of the simulations for an intermediate case $\left(\rho_{i} \approx 4 \rho_{e}\right.$ and $\left.\xi_{i} / \xi_{e} \approx 4\right)$ are shown in Fig.6. Due to the different gyration radii charge separation occurs in regions where a density gradient exists. Thus, an electric field arises which is directed inward, preventing the outward motion of the positively charged particles $(i)$ with their larger gyration radius. A large deviation of $n_{i}(r)$ from $n_{e}(r)$ is not allowed (Fig.6) but also not necessary, since already a small charge imbalance inside the plasma radius $\left(r_{p l}=0.02\right.$ $m)$ is sufficient to generate an electric field of about $E=-1300 \mathrm{~V} / \mathrm{m}$ as obtained in the simulation at position $r_{p l}$ (see Fig.6).

The required averaged charge imbalance $n_{q}=\left\langle n_{i}-n_{e}\right\rangle$ can be readily estimated using Gauss's theorem

$$
\int_{V} \operatorname{div} \boldsymbol{E} d V=\int_{V} \frac{e\left(n_{i}-n_{e}\right)}{\epsilon_{o}} d V=\oint \boldsymbol{E} d \boldsymbol{S}
$$

which leads to $e n_{q} \pi r_{p l}^{2} \Delta z / \epsilon_{o}=E_{r} 2 \pi r_{p l} \Delta z$ and following

$$
n_{q}=\frac{\left.2 \epsilon_{o} E_{r}\right|_{r=r_{p l}}}{e r_{p l}}=-\frac{2 \epsilon_{o} 1300 \mathrm{~V} / \mathrm{m}}{e 0.02 \mathrm{~m}}=-7 \times 10^{12} \mathrm{~m}^{-3}
$$

This excess of 'e'-particles inside $r_{p l}$ is two orders of magnitude smaller than the plasma density in this calculations $\left(n_{p l}=10^{15} \mathrm{~m}^{-3}\right)$.

In the case of small gyration radii in comparison with the density decay length $\left(\rho_{i}, \rho_{e} \ll\right.$ $\lambda_{n}$ ) the mean rotation velocities of both species can be represented as the sum of the diamagnetic drift velocity and the $(\boldsymbol{E} \times \boldsymbol{B})$-drift velocity

$$
v_{\theta}^{e, i}=\frac{E \times B}{B^{2}}+\frac{B \times(\nabla p)}{n q_{e, i} B^{2}}
$$

which gives

$$
v_{\theta}^{i}(r)=-\frac{E(r)}{B_{z}}+\frac{\mathrm{k} T_{i}}{e n_{i}(r) B_{z}} \frac{\partial n_{i}(r)}{\partial r}, \quad v_{\theta}^{e}(r)=-\frac{E(r)}{B_{z}}-\frac{\mathrm{k} T_{e}}{e n_{e}(r) B_{z}} \frac{\partial n_{e}(r)}{\partial r}
$$



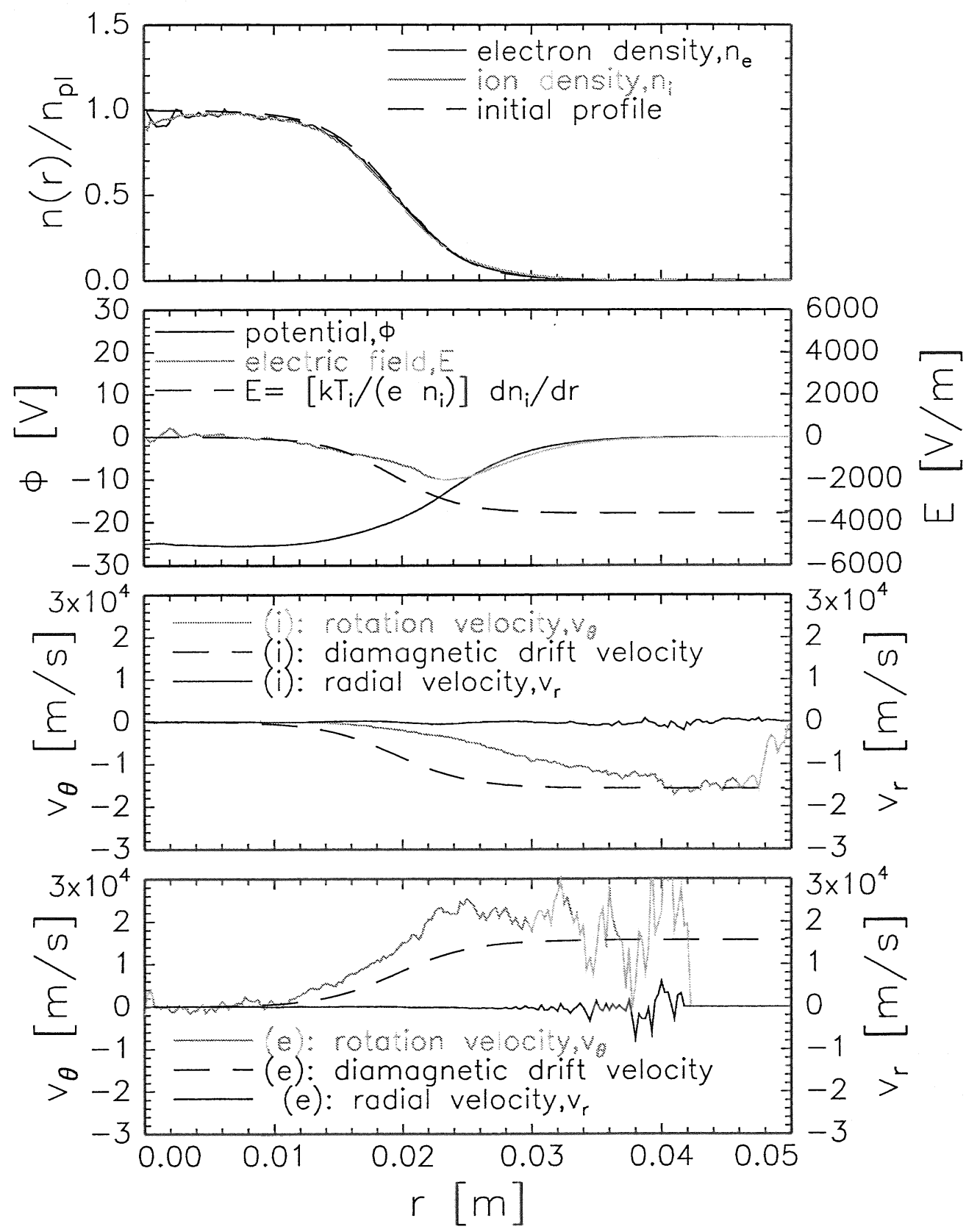

Figure 6: Mean radial profiles of $n, E, \phi, v_{r}$ and $v_{\theta}$ for both species $e$ and $i$. (parameters: $R=0.05 \mathrm{~m}, n_{p l}=10^{15} \mathrm{~m}^{-3}, B=0.22 \mathrm{~T}, T_{e}=T_{i}=10 \mathrm{eV}, m_{i}=4, m_{e}=0.2 \mathrm{amu}$, $\left.\xi_{i}=4, \xi_{e}=0.9, \rho_{i}=3, \rho_{e}=0.7 \mathrm{~mm}\right)$ 
The $(\boldsymbol{E} \times \boldsymbol{B})$-drift velocity is equal for both species. Hence, the rotation velocity of the 'e'-particles is enhanced due to the action of the radial electric field, but the 'i'-particles are slowed down (Fig.6).

The resulting angular momentum of species ' $\mathrm{i}$ ' is a factor of two smaller than $L_{i}^{\text {diam }}=$ $-7.8 \times 10^{-13} \mathrm{~kg} \mathrm{~m}^{2} / \mathrm{s}$ (Fig.7) and $L_{e} \approx 2 \times L_{e}^{\text {diam }}$. As already decribed in Sec. 3.2 the
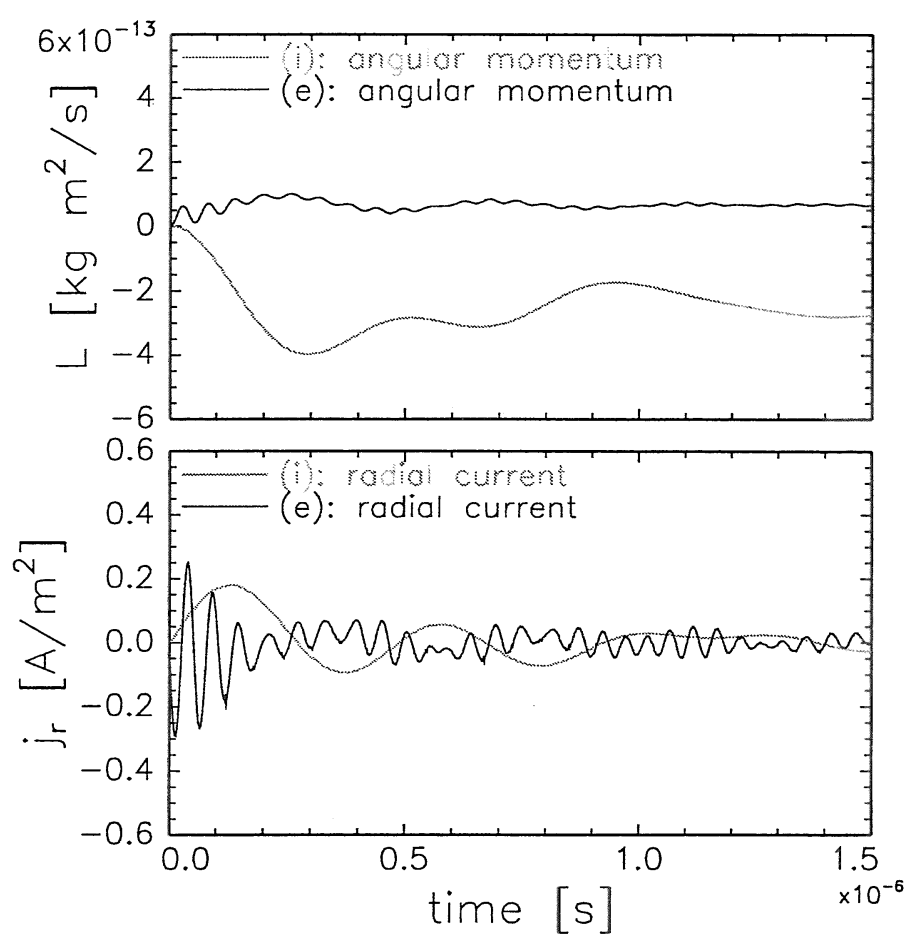

Figure 7: Temporal behavior of $L_{e, i}$ and $j_{r}^{e, i}=$ $q n_{e, i} v_{r}^{e, i}$. The latter value has been averaged over the plasma volume for each time step. (parameters as given in Fig.6, gyration time $\tau_{\rho, i}=1.2 \times 10^{-6}$ and $\tau_{\rho, e}=$ $\left.6 \times 10^{-8} s\right)$

$\boldsymbol{j}^{e, i} \times \boldsymbol{B}$-force provides the torque resulting in a change of the angular momentum, but it acts independently for each species, which was found in the calculations. This means, that there is no transfer of momentum from one species to the other. The oscillation of the radial current $j_{r}^{e}$ is characterized by the corresponding gyration frequency $f_{e}=2 \pi / \tau_{\rho, e}$, but this is not the case for species ' $\mathrm{i}$ ', where $j_{r}^{i}$ changes with a higher frequency than $f_{i}=2 \pi / \tau_{\rho, i}$.

\subsection{Case $\xi_{i} \gg \xi_{e}$}

Taking electrons as species 'e' the following conditions can be fulfilled:

$$
\rho_{e} \ll \rho_{i}, \quad \rho_{i} \ll \lambda_{n}=n|d n / d r|^{-1}, \quad \xi_{e} \ll \xi_{i}
$$

Due to their small mass and subsequently small gyration radii the electrons are tied strongly to the magnetic field lines. In order to save computer time the motion of the electrons is determined by the diamagnetic and $(\boldsymbol{E} \times \boldsymbol{B})$-drift only. The results of such calculations for the parameter region (22) are shown in Fig.8. As seen the density profiles 


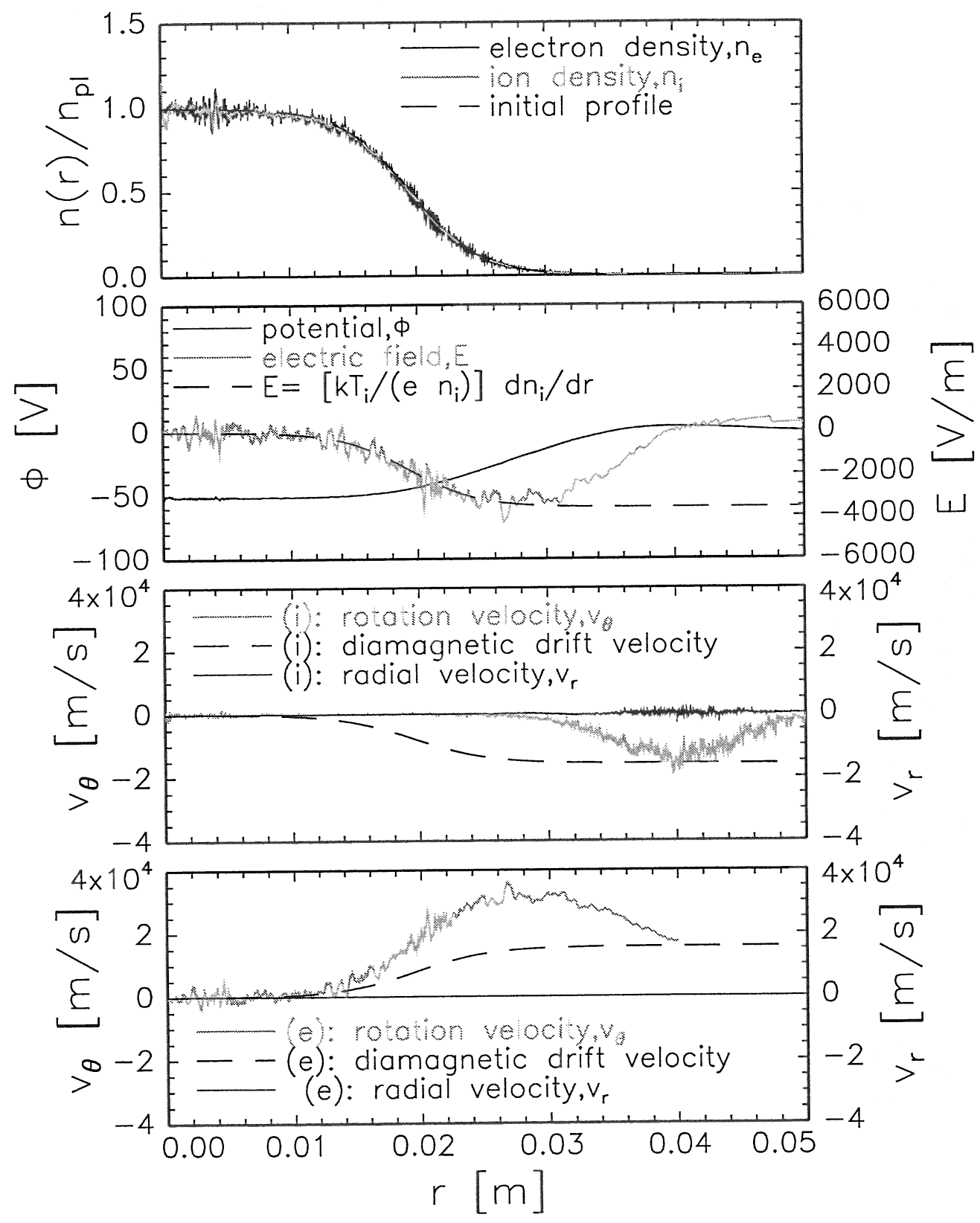

Figure 8: Mean radial profiles of $n, E, \phi, v_{r}$ and $v_{\theta}$ for both species $e$ and $i$. (parameters: $R=0.05 \mathrm{~m}, n_{p l}=10^{17} \mathrm{~m}^{-3}, B=0.22 \mathrm{~T}, T_{e}=T_{i}=10 \mathrm{eV}, m_{i}=1 \mathrm{amu}, m_{e}=9.1 \times 10^{-31}$ $k g, \xi_{i}=20, \xi_{e}=0.5, \rho_{i}=1.5, \rho_{e}=0.035 \mathrm{~mm}$ ) 
$n_{e}(r)$ and $n_{i}(r)$ are almost equal. The remaining small charge imbalance which is too small to be distinguished visually in the figure leads to occurence of a radial electric field. In the region $r<0.03 m$ this electric field is equal to

$$
E(r)=\frac{\mathrm{k} T_{i}}{e n_{i}(r)} \frac{\partial n_{i}(r)}{\partial r}
$$

since the force due to the ion pressure should be balanced by the electric field force at each radial position

$$
e n_{i}(r) E(r)=\nabla p_{i}=\mathrm{k} T_{i} \frac{\partial n_{i}(r)}{\partial r} .
$$

Note, that this relation holds only at radial positions where the mean rotation velocity of the ions is zero (Fig.8). In this rather large region $(0<r<0.03 m)$ which extends over almost the whole plasma column the ion diamagnetic drift is exactly cancelled by the $(\boldsymbol{E} \times \boldsymbol{B})$-drift. The electrons, on the other hand, double their rotation speed in this region (for the considered case of $T_{e}=T_{i}$, Fig.8).

With other words, there is no time left for the ions to establish their diamagnetic drift during one gyration time, since this is prevented by the arising electric field on a very much shorter time scale going with the plasma frequency.

\subsubsection{Ion trajectories}

Without an electric field ions move in magnetic fields on circular orbits. For illustration, four ion trajectories are shown in Fig.9, where the ions are started from the same position in different directions.

With an electric field $E_{x}\left(E_{y}=E_{z}=0\right)$ the ions describe cycloidal trajectories due to the additional $(\boldsymbol{E} \times \boldsymbol{B})$-drift, where the shape of the trajectories depends on the starting direction. Further, the resulting density distribution of ions started at the same position $x_{s t}$ with a Maxwellian velocity distribution is changed due to the action of this electric field. Note, that the maximum of the distribution remains at the starting position, but its center of gravity is shifted by [7]

$$
\Delta x=\frac{v_{d}}{\omega_{i}}=\frac{E_{x} m_{i}}{e B_{z}^{2}}=-0.00044 m
$$

( $v_{d}$ is the $(\boldsymbol{E} \times \boldsymbol{B})$-drift velocity and $\omega_{i}$ is the ion gyration freqency) which was proved by integrating $\Delta x=\int x n(x) d x / \int n(x) d x-x_{s t}$. This inward shift compensates the local outward shift $\Delta x_{\rho}$ caused by the 'broadening' of the density profile due to finite ion gyro radii (see in Fig.3).

$$
\Delta x_{\rho}=\rho^{2} / \lambda_{n}
$$

with the density decay length $\lambda_{n}=n|d n / d r|^{-1}$, so that $\Delta x+\Delta x_{\rho}=0$. 

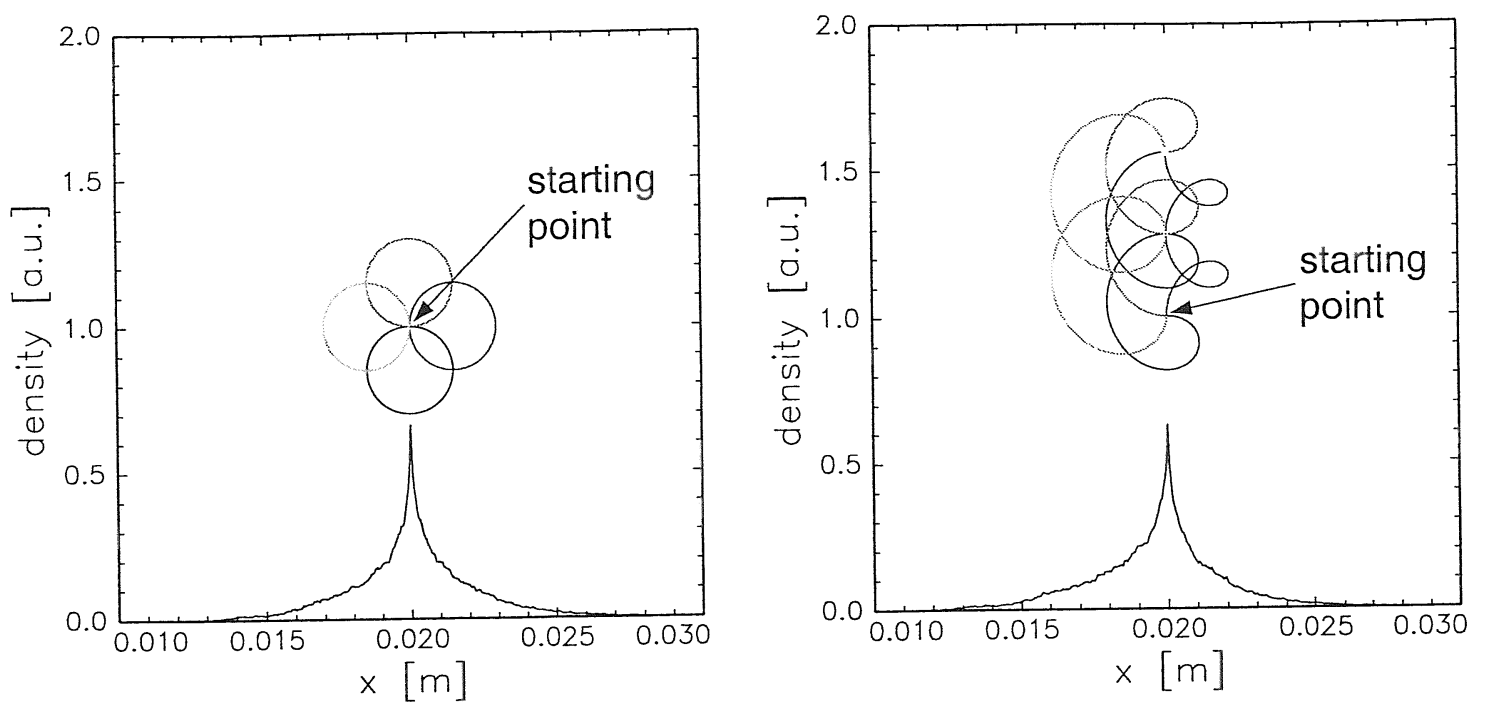

Figure 9: Density distributions of ions started at a fixed position $x_{s t}=0.02 \mathrm{~m}$ without (left figure) and with an electric field (right figure) $E_{x}=-2000 \mathrm{~V} / \mathrm{m}$. The magnetic field is directed out of the plane toward the reader. The trajectories of four ions are shown. (parameters: $B=0.22 \mathrm{~T}, T_{i}=10 \mathrm{eV}, m_{i}=1 \mathrm{amu}$ ). Note, that after each time period all particles meet again in one point.

\section{4 'Electric' confinement of ions}

In the case of $\xi_{i}>30$ the ions are confined in a plasma column by a radial electric field. Ion confinement means in this sense that the ion density profile should almost be equal to the electron density profile, since large deviations are not allowed. The initial diamagnetic drift of the ions is exactly cancelled and the resulting mean rotation velocity is zero, i.e. the term $\left(\boldsymbol{j}_{i} \times \boldsymbol{B}\right)$ also vanishes in the global force balance. Considering the global behavior of the ion the effect of the magnetic field is therefore negligible and the approximations

$$
n_{i}(r)=c_{n} n_{p l} \exp \left(-\frac{e \phi(r)}{\mathrm{k} T_{i}}\right)
$$

or

$$
\phi(r)=-\frac{\mathrm{k} T_{i}}{e} \ln \left[\frac{n_{i}(r)}{c_{n} n_{p l}}\right]
$$

can be used (see in [8] and [1]) and are valid for a Maxwellian velocity distribution under steady state conditions. The constant $c_{n}$ is determined by the plasma neutrality condition

$$
\int_{0}^{R}\left[n_{i}(r)-n_{e}(r)\right] r d r=0
$$

Substituting for $\phi(r)$ and $n_{i}(r)$ the calculated values of the ion density $n_{i}(r)$ and potential $\phi(r)$ (as shown in Fig.8) the validity of the relations (27-29) is demonstrated in Fig.10. 
Good agreement could be achieved using $c_{n}=0.0059$, where deviations occur only in the outer region of $r>0.03 \mathrm{~m}$.

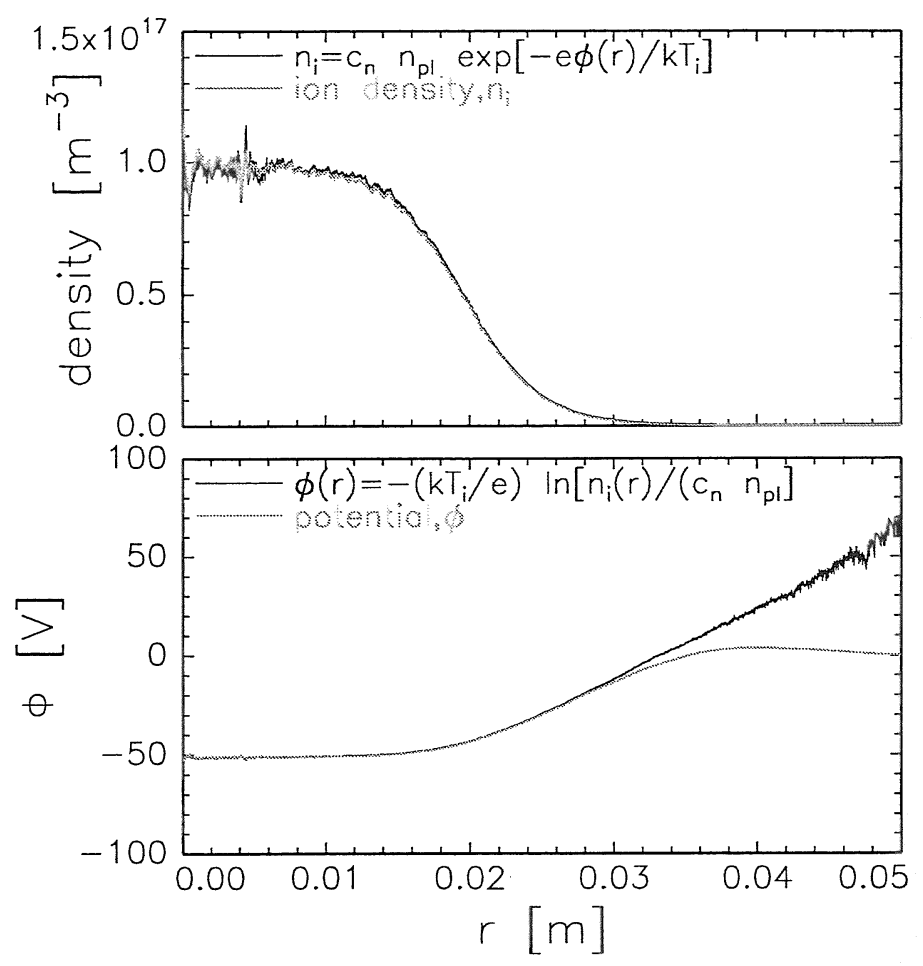

Figure 10: Comparison of the calculated density and potential profiles (from Fig.8) with the analytical results according to Eqs. $(27,28)$ with $c_{n}=0.0059$.

It is interesting to check in which region of plasma parameters such a simple description of the ion density profile (Eg.(27)) can be applied. Rearranging (1) a 'critical' value of the magnetic field $B$ can be presented as a function of the plasma density

$$
B^{c r i t}=\frac{1}{\xi_{e, i}^{c r i t}} \sqrt{\frac{n_{p l} m_{e, i}}{\epsilon_{o}}}
$$

This value has been calculated in a wide range of $n_{p l}$ for both plasma species using two fixed values of $\xi^{\text {crit }}=1$ and $\xi^{\text {crit }}=30$ (Fig.11). The parameter regions of interest $\left(B, n_{p l}\right)$ for plasma and fusion experiments are indicated in Fig.11 and are located beneath the line corresponding to deuterium plasma ions and $\xi_{i}=30$ but above the line regarding to the electrons. For almost all plasma conditions met in plasma experiments the parameter $\xi$ for electrons is about unity, the respective parameter for deuterium ions is larger than 30. This means, that in contrast to the electrons the plasma ions are only insignificantly affected by the magnetic field and thus the approximation (27) can be used.

Substituting for $n_{i}(r)$ from (27), the Poisson equation gives

$$
\frac{\partial^{2} \phi}{\partial r^{2}}+\frac{1}{r} \frac{\partial \phi}{\partial r}+\frac{e c_{n} n_{p l}}{\epsilon_{o}} \exp \left(-\frac{e \phi}{\mathrm{k} T_{i}}\right)=\frac{e n_{e}(r)}{\epsilon_{o}}
$$




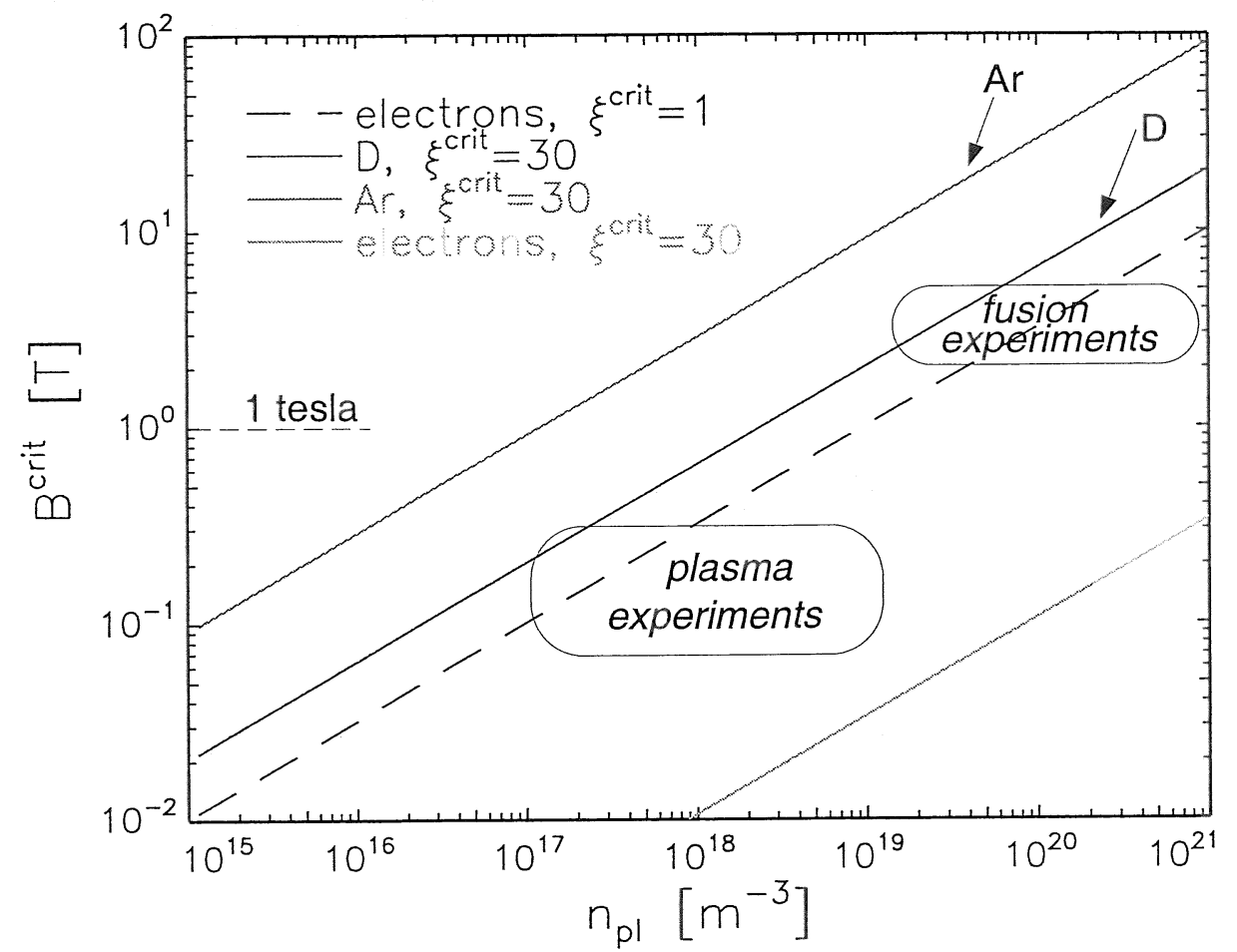

Figure 11: $B^{\text {crit }}$ vs plasma density $n_{p l}$ calculated for electrons, deuterium and argon ions.

where $n_{p l}$ denotes the electron density at $r=0$, i.e. $n_{e}(0)=n_{p l}$. This non-linear differential equation together with the boundary conditions $\partial \phi(r=0) / \partial r=0$ and $\phi(r=$ $R)=0$ can be solved by iterations. Excellent convergence is achieved by applying in addition the method of successive underrelaxation. For this a linear combination of the old value and the value given by the new step is taken, so that

$$
\phi^{\text {new }}=\eta \phi^{\text {calc }}+(1-\eta) \phi^{\text {old }}
$$

Underrelaxation means $\eta<1$. During each iteration step the ion density profile is normalized, i.e. the constant $c_{n}$ is obtained according to Eq.(29),

Taking for the ion temperature a value of $\mathrm{k} T_{i}=2 \mathrm{eV}$ the profiles of the ion density have been calculated for different plasma densities (Fig.12). The corresponding profiles of the potential and the electric field are shown in Fig.13. With increasing plasma density the profiles of $n_{e}$ and $n_{i}$ become almost equal (Fig.12). The remaining small charge difference establishs a negative electric field which holds the ions inside the plasma column. At higher densities the electric field at the plasma boundary converges to its maximum value (Table 1). 


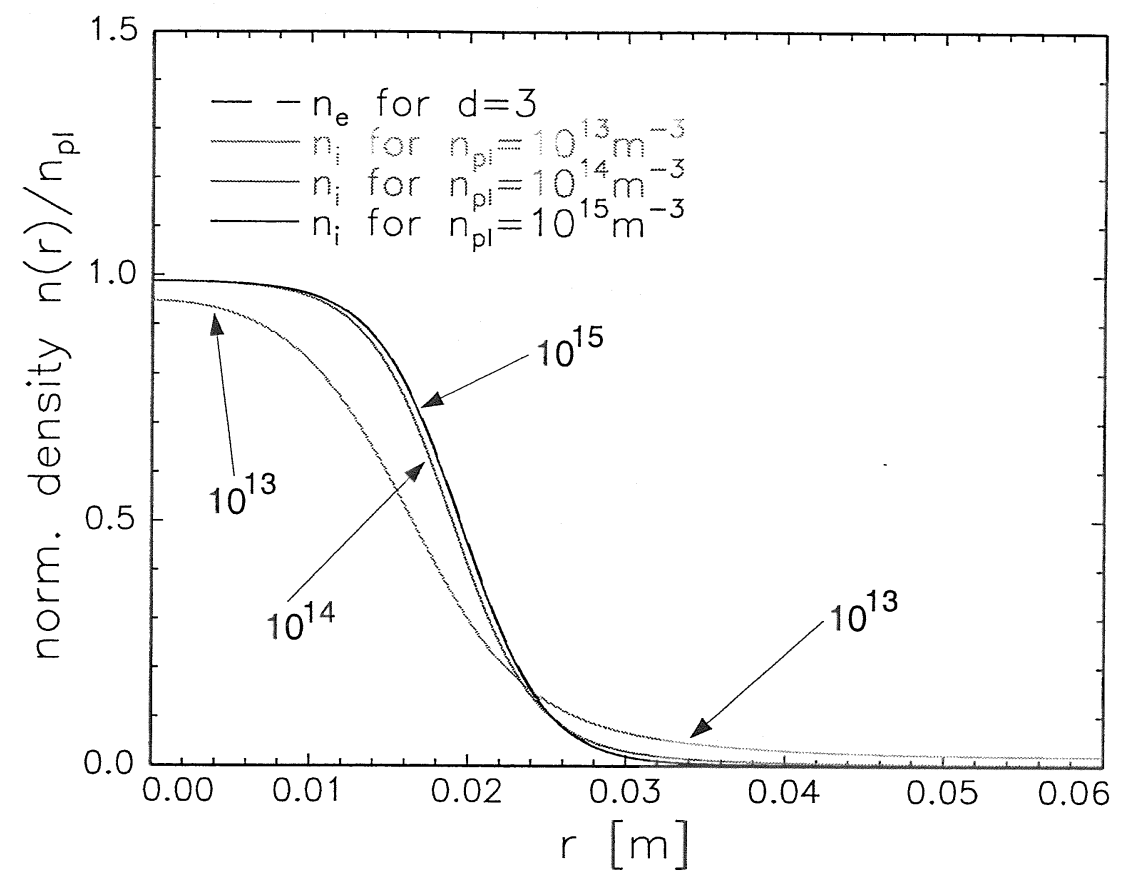

Figure 12: Normalized profiles of ion density varying the plasma density $n_{p l}=$ $10^{13}, 10^{14}, 10^{15} \mathrm{~m}^{-3}$. The electron profile is obtained using Eq.(10) with $d=\sqrt{D_{\perp} t}=3$ $m m\left(r_{p l}=0.02 m, R=0.06 m\right.$, case of $\left.\chi_{i}>1000\right)$.

Table 1: Calculated electric field $E_{r}$ at position $r=r_{p l}=0.02 \mathrm{~m}$ as function of plasma density $n_{p l}$ and diffusion length $d\left(\mathrm{k} T_{i}=2 \mathrm{eV}\right)$.

\begin{tabular}{|c|c|c|c|c|c|c|}
\hline$d=\sqrt{D_{\perp} t}$ & $n_{p l}=10^{13}\left[\mathrm{~m}^{-3}\right]$ & $10^{14}$ & $10^{15}$ & $10^{16}$ & $10^{17}$ & $10^{18}$ \\
\hline \hline $1 \mathrm{~mm}$ & $E_{r}=-465 \mathrm{~V} / \mathrm{m}$ & -998 & -1280 & -1228 & -1219 & -1218 \\
\hline $3 \mathrm{~mm}$ & $E_{r}=-327 \mathrm{~V} / \mathrm{m}$ & -434 & -409 & -405 & -405 & -405 \\
\hline $10 \mathrm{~mm}$ & $E_{r}=-135 \mathrm{~V} / \mathrm{m}$ & -78 & -128 & -127 & -127 & -127 \\
\hline
\end{tabular}

A stronger electric field is required if the ion temperature $T_{i}$ increases (Fig.14). As already mentioned in Sec. 3.4, instead of the numerical solution of Eqs. $(29,31)$ the same result can be obtained from the simple force balance

$$
E(r)=\frac{\mathrm{k} T_{i}}{e n_{i}(r)} \frac{\partial n_{i}(r)}{\partial r} \text { and } \phi(r)=\int_{r}^{R} E(r) d r
$$

However, usually the exact ion density profile is unknown - without solving Eq.(31). Nevertheless, quite accurate estimations (at least for $E$ ) are possible by taking the electron density profile instead. Results of such calculations are shown in Fig.14 (dashed lines). Deviations occur again in the outer region since the use of relation (33) by replacing $n_{i}(r)$ with $n_{e}(r)$ gives exact results only for $n_{p l} \rightarrow \infty$. 


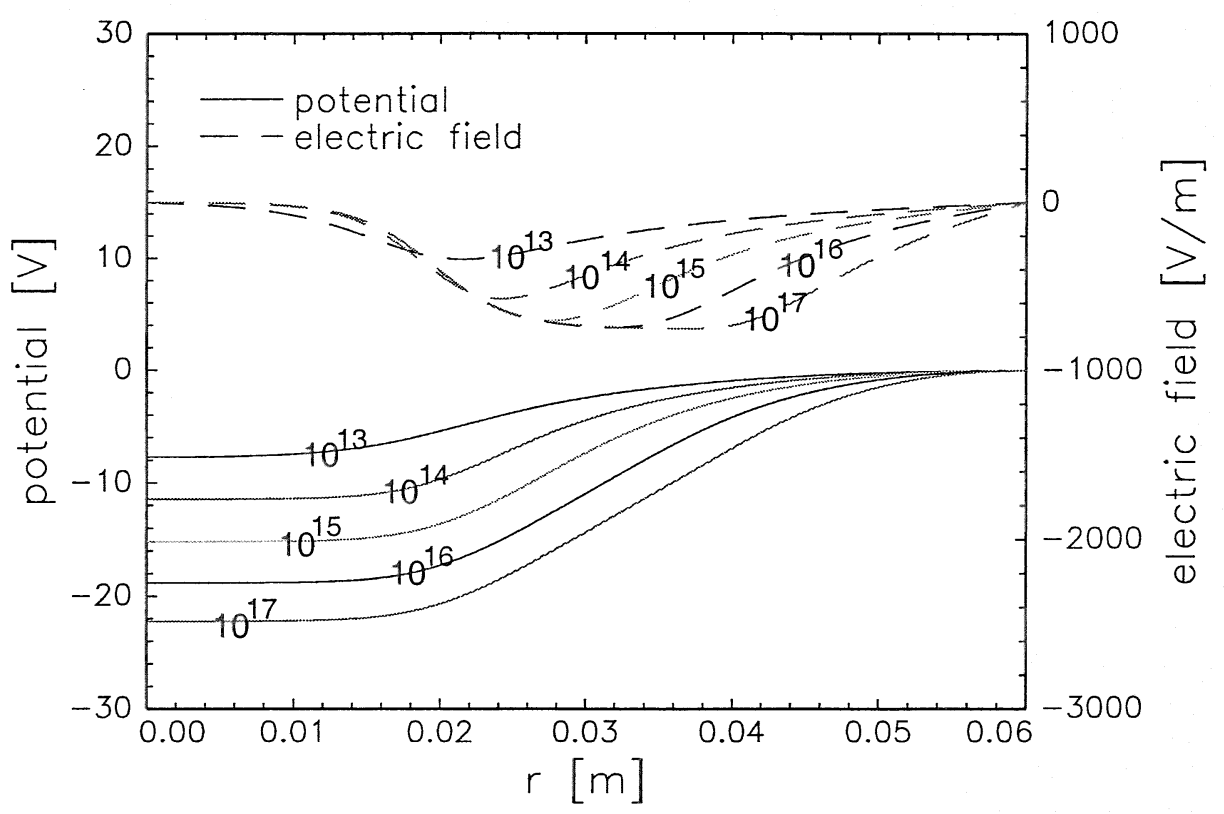

Figure 13: Electric field and potential distributions with the plasma density $\left(n_{p l}=10^{13}-\right.$ $\left.10^{17} \mathrm{~m}^{-3}\right)$ as parameter $\left(d=\sqrt{D_{\perp} t}=3 \mathrm{~mm}, \mathrm{k} T_{i}=2 \mathrm{eV}\right)$.

\section{Discussion and Summary}

There is one dimensionless ratio

$$
\chi=\frac{n_{p l} m}{\epsilon_{o} B^{2}}
$$

connecting three parameters: the plasma density $n_{p l}$, the mass of considered species $m$ and the magnetic field strength $B$, which characterizes the behavior of a magnetized plasma. It determines the processes of charge shielding (Sec. A), polarization (Sec. C), confinement (Sec. 3,4) and wave propagation since this parameter $\chi$ can also be presented as

$$
\chi=\xi^{2}=\frac{\rho^{2}}{\lambda_{D}^{2}}=\frac{j_{p o l}}{\epsilon_{o} \partial E / \partial t}=c^{2} \frac{\text { Density }}{\text { Tension }}=\frac{c^{2}}{v_{A}^{2}}
$$

where $c=1 / \sqrt{\epsilon_{o} \mu_{o}}$ is the speed of light. The velocity $v_{A}=\sqrt{B^{2} /\left(\mu_{o} M n_{p l}\right)}$ is known as the Alvén velocity and describes the propagation of transverse waves in analogy with the vibrations of mass-loaded elastic strings under tension $B^{2} / \mu_{o}$ [8]. Further, this ratio is the main parameter in the analysis of the complex phenomena in the electric sheath near a plasma-material interface.

Despite of the large variety of plasma devices in many experiments the corresponding parameter $\chi$ for the electrons is about unity and $\chi$ for the plasma ions (usually hydrogen or deuterium) is larger than $1000\left(\xi_{i}>30-40\right)$. As shown in Sec. 4 this is a fortunate situation since then a very simple description is possible instead of time-consuming computer simulations. The point is, that in this case the global behavior of the ions is 


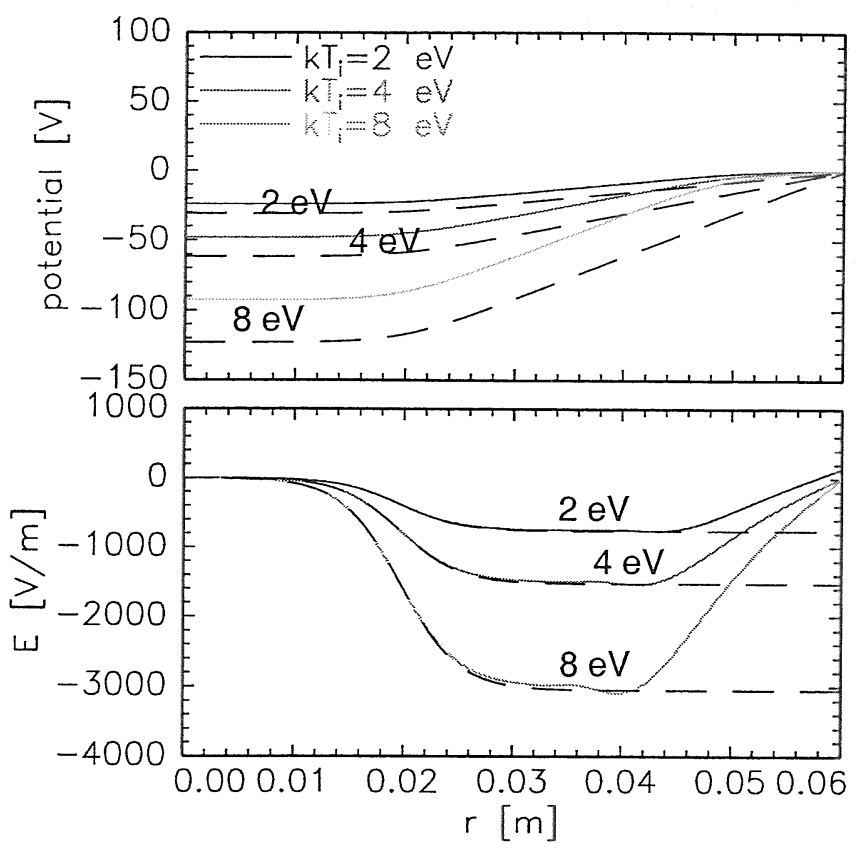

Figure 14: Electric field and potential distributions for $d=$ $\sqrt{D_{\perp} t}=3$ and different $\mathrm{k} T_{i}$ but the same $n_{p l}=10^{18} \mathrm{~m}^{-3}$ in comparison with the analytical results (dashed lines, Eq.(33)).

determined only by an electric field arising due to charge separation in regions with a pressure gradient (Sec. 3). In this process a tiny fraction of the kinetic energy of the plasma is converted to potential energy of the electric field.

The force balance for the ions at a certain radial position in the plasma colum is given by

$$
n_{i} e E=\nabla p_{i}
$$

and for the electrons

$$
-n_{e} e E-n_{e} e\left[v_{\theta}^{e} \times B_{z}\right]=\nabla p_{e}
$$

(note $E(r)<0$ and $v_{\theta}^{e}>0$ ). Substituting for $E$ from Eq.(36) yields

$$
v_{\theta}^{e}(r)=-\frac{\nabla p_{i}}{e n_{i}(r) B_{z}}-\frac{\nabla p_{e}}{e n_{e}(r) B_{z}}
$$

This leads to the same relation $j \times B=\nabla p$ as derived in the simplified MHD theory, but the current is supplied by the electrons only and is not the sum of the electron and ion diamagnetic currents.

In the MHD theory (see in [9]) the terms with $n_{q}(r)=n_{i}(r)-n_{e}(r)$ are cancelled, but the electric field remains in the equations. For example, by having additional informations about the ion rotation velocity the correct electric field can be deduced using the ion momentum equation. With known electric field the charge imbalance $n_{q}(r)$ can then be estimated using the Poisson equation. However, by considering steady-state situations it is not possible to distinguish whether there is an electric field or a corresponding rotation velocity or both. 
The advantage of PIC simulations is demonstrated in this paper since all important parameters such as $n_{q}, E, v_{\theta}^{i}$ and $v_{\theta}^{e}$ have been self-consistently obtained (for different ratios $\rho_{i} / \rho_{e}$ ) by modeling the time evolution of the plasma. It is shown, that for the same starting conditions (zero plasma momentum), for the same boundary conditions and using the same numerical solution strongly different results are obtained by changing only the ratio $\rho_{i} / \rho_{e}$. It is verified in the simulations, that the observed change of momentum is caused by radial currents supplying a torque.

The charge imbalance is indeed small, many orders of magnitude smaller than the plasma density, but sufficiently large to generate the electric field confining the plasma ions. The motion of single ions is still strongly affected also by the magnetic forces, but the ions act in their global behavior independently of the magnetic field in the case of $\chi_{i}>1000$ and one could speak of 'electric ion confinement'. Only in the case of equal gyration radii of both species the usual inward $\boldsymbol{j} \times \boldsymbol{B}$-force due to the diamagnetic currents of both species balances the outward force from the pressure gradient. 


\section{A Appendix: Charge shielding}

The reaction of a plasma on a imbedded test charge is a fundamental problem in plasma physics. The distance over which the field from such a charge is shielded out is the Debye length which was first derived in the theory of electrolytes by Debye and Hückel. The assumption that the electrostatic potential energy is much less than the mean thermal energy, is part of the commonly known derivation (e.g. see in [8]) of the Debye length

$$
\lambda_{D}=\sqrt{\frac{\epsilon_{o} \mathrm{k} T_{e} \mathrm{k} T_{i}}{n_{p l} e^{2}\left(\mathrm{k} T_{e}+\mathrm{k} T_{i}\right)}}
$$

(see in [10] for a more detailed description, e.g. considering also the effect of fast moving particle). Note, that the smaller temperature of both species defines $\lambda_{D}$. In particular, ideal shielding $\left(\lambda_{D} \rightarrow 0\right)$ occurs in a cold ion plasma $\left(T_{i} \simeq 0\right)$.

In order to describe the effect of an external magnetic field on the shielding behaviour of a plasma it is sufficient to consider a plasma box of length $L$. This means that all particles are started in the simulation uniformly distributed along the axis $x$ with velocity components given by (2). Periodic boundary conditions for the potential $\left(\phi_{x=0}=\phi_{x=L}\right)$ are applied as well as for the particles, i.e. particles leaving the calculation region at one side $(x=0$ or $x=L)$ are set back to the other side. The electric field $E(x)$ (onedimensional in the $x$-direction) is determined by

$$
E_{x}=-\frac{\partial \phi}{\partial x}, \quad \nabla^{2} \phi=\frac{\partial^{2} \phi}{\partial x^{2}}=\frac{e}{\epsilon_{o}}\left[n_{e}(x)-n_{i}(x)\right]
$$

Since the assumed magnetic field has only a $B_{z}$-component, the particles gyrate on the $(x, y)$-plane.

In the case of a strong magnetic field the particles are tied to the magnetic field lines. Their motion across $B$ is restricted. Simulations have been performed for different values of $\xi=\rho / \lambda_{D}$ which is the ratio of the gyration radius $\rho$ to the Debye length. The masses and the temperatures of both species are taken equal, only the magnetic field $B_{z}=B$ is changed. Fig. 15 shows the potential distribution if a test charge of $Q_{s}=5 \times 10^{10}$ ions $/ \mathrm{m}^{2}$ (given as a surface charge) is immersed in the center of the calculation region. The corresponding density distributions are shown in Fig.16. These are the results of a simulation using the following parameters: $L=0.02 \mathrm{~m}, n_{p l}=10^{14} \mathrm{~m}^{-3}, T_{e}=T_{i}=2 \mathrm{eV}$, $m_{e}=m_{i}=1 \mathrm{amu}$ (this choice of parameters serves simply as an example).

Charge neutrality in the analyzed plasma region is ensured in the simulation, i.e. the sum of the positive field charges, represented by the particles of species $i$, and the positive test charge balance the negative field charges (species $e$ ). The dimension of the plasma $L$ has been changed in the simulations to keep the grid step $\Delta x$ always smaller than the Debye length. According to the Courant-Friedrichs-Lewy criterion (see Sec. 2) the timestep $\Delta t$ has been changed as well. Further, a plasma which is disturbed at a certain time moment 


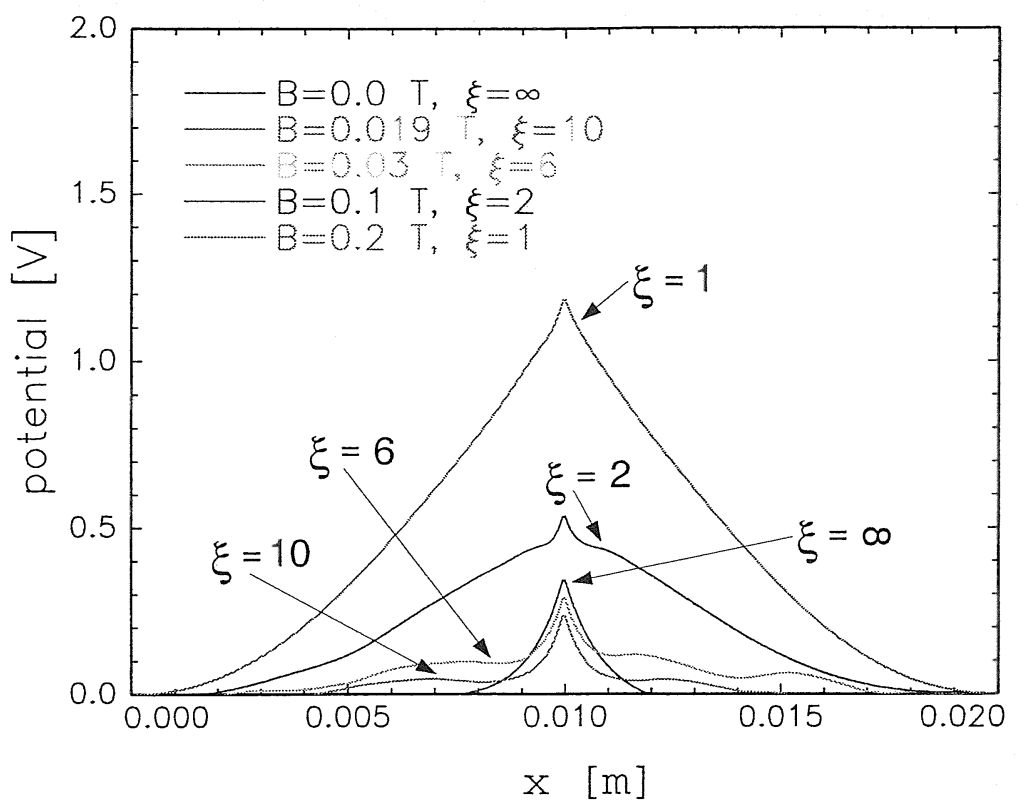

Figure 15: Potential distributions in the vicinity of a surface test charge for $n_{p l}=$ $10^{14} \mathrm{~m}^{-3}$ and different $\xi$. (parameters: $L=0.02 \mathrm{~m}$, $T_{e}=T_{i}=2 \mathrm{eV}, m_{i}=m_{e}=$ 1 amu)

usually starts to oscillate. This behaviour is observed also in the modeling, so that the presented results are obtained by averaging over time.

For $\rho \leq \lambda_{D}$ (i.e. $\xi \leq 1$ ) the plasma can not shield the perturbing charge (Fig.15 and Fig.16, the different values of $B$ and $\xi$ are indicated in the figures). In this case, the potential distribution is mainly defined by the test charge and the boundary conditions and becomes similar to the potential distribution corresponding to a situation where only the test charge $Q_{s}$ is located in the center of the region (i.e. without plasma)

$$
\phi(x)=\left\{\begin{aligned}
Q_{s} x /\left(2 \epsilon_{o}\right) & \text { for } \quad 0<x \leq L / 2 \\
Q_{s} L[1-(2 x-L) / L] /\left(4 \epsilon_{o}\right) & \text { for } \quad L / 2<x \leq L
\end{aligned}\right.
$$

For small values of $\xi \leq 1$, i.e. strong magnetic field, the gyrating charged particles are not able to overcome the magnetic field force and remain at their initial positions (Fig.16, $\xi=0.2$ ). Thus, the particles surrounding the test charge cannot balance it as they do in the magnetic-field-free case (Fig.16, $\xi=\infty$ ).

On the other hand, for $\rho>10 \lambda_{D}$, the magnetic field affects only marginally the motion of the charged particles and the potential distribution is almost equal to the magnetic-fieldfree case $(\xi=\infty)$ where the potential decays with the Debye length (here $\lambda_{D}=7.4 \times 10^{-4}$ $m)$.

The next question to be addressed is what happens if the mass of one species is much smaller than the mass of the other species, e.g. $m_{e} \ll m_{i}$. The lighter species is strongly bound to the magnetic field lines in case of small $\xi_{e}$, but the heavier species might not if its corresponding parameter $\xi_{i}$ is much larger $\left(\xi_{i} / \xi_{e} \geq 10\right)$ under the same conditions $\left(n_{p l}, B\right)$. Since a compensation of the test charge is possible by acting of only one species, the behaviour of the plasma is determined by the heavier particles, which are less affected 


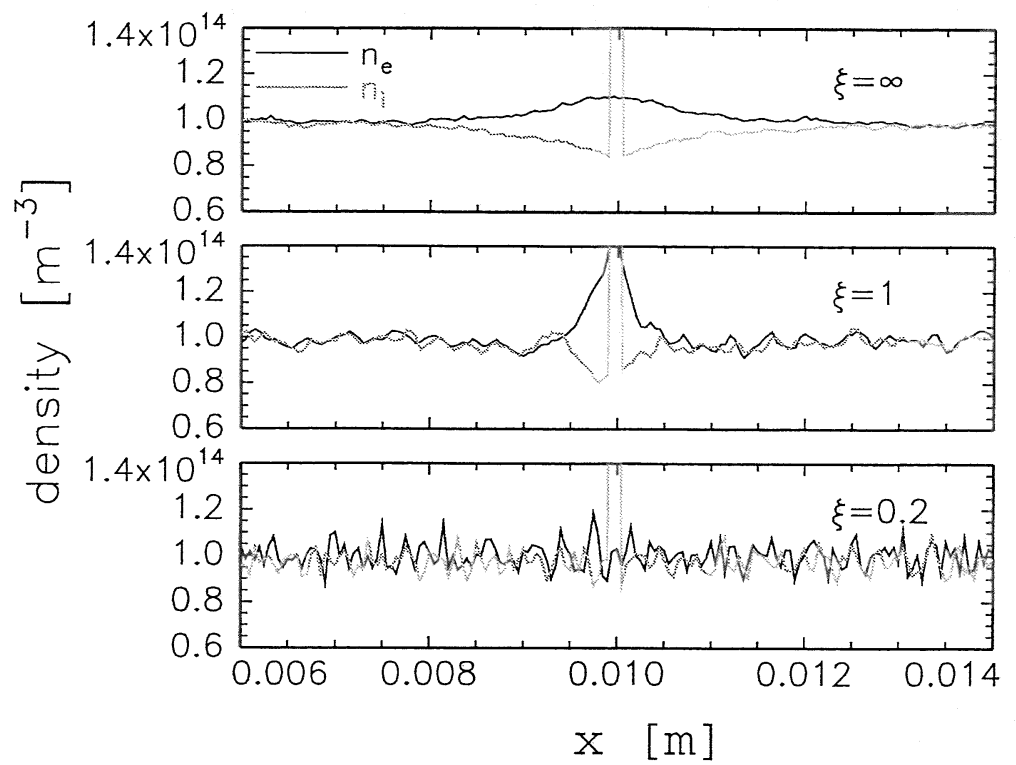

Figure 16: Distributions of the densities $\left(n_{e, i}\right)$ for different $\xi=0.2,1, \infty$ as indicated in the figure. Same parameters as in Fig.15.

by the magnetic field. These particles are able to shield the test charge (if $\xi_{i} \geq 10$ ) and the same strong decay of the potential characterized by $\lambda_{D}$ is observed as in the case of $\xi_{i}=\xi_{e} \geq 10$. A more detailed description of the shielding problem in magnetized plasmas is given in [1]. 


\section{B Appendix: Scaling rules}

Since the Debye length is the natural scaling length of the charge shielding problem, the potential distributions do not change, for example, if the plasma density is varied but the normalized system length $L / \lambda_{D}$ remains the same. The results of such calculations similar to those described in Sec. A are shown in Fig.17. The only differences between

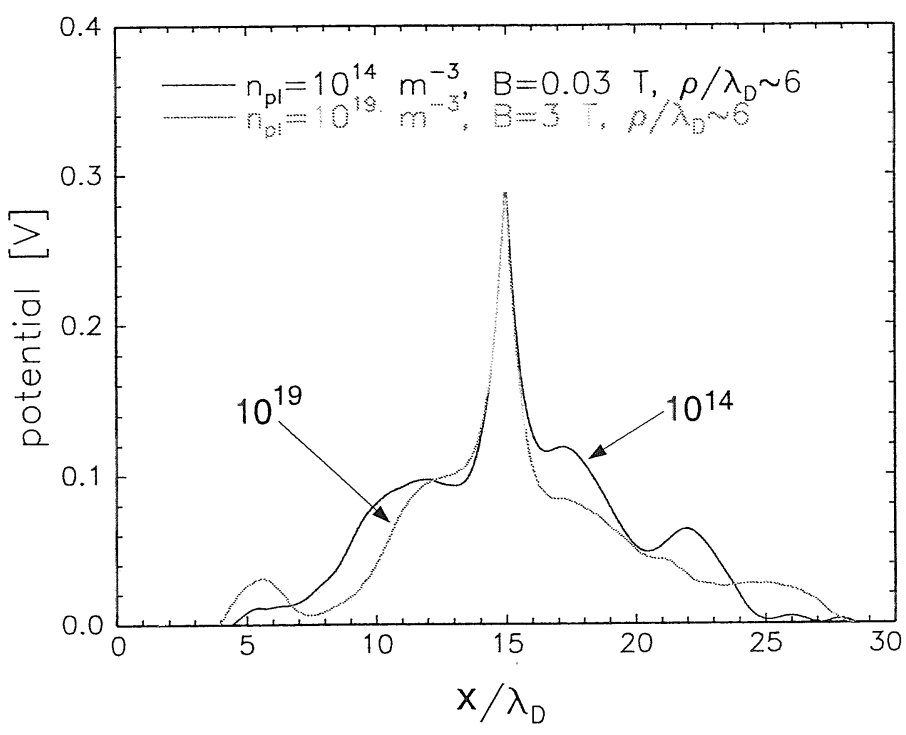

Figure 17: Potential distributions vs normalized length $x / \lambda_{D}$ for two different densities $\left(n_{p l}=10^{14}, 10^{19} \mathrm{~m}^{-3}\right)$ but equal value of $\xi(\simeq 6)$. (parameters: $T_{e}=T_{i}=2$ $\left.\mathrm{eV}, m_{i}=m_{e}=1 \mathrm{amu}\right)$

the two curves, occuring at larger distances from the test charge (Fig.17), are caused by statistical errors. Obviously, the electric field itself depends on $n_{p l}$, but the product $E \cdot \lambda_{D}$ does not. Note, that the total number of charged particles is not equal for both cases, only the normalized box length $L / \lambda_{D}$ is the same.

Keeping the plasma geometry (for example the width of the plasma region), the mass and the charge of the plasma species unchanged the following scaling rules can be applied by comparing two different sets of plasma parameters. The conditions

$$
n_{p l}^{\prime}=f_{n} \cdot n_{p l}, \quad T_{e, i}^{\prime}=f_{n} \cdot T_{e, i}, \quad B^{\prime}=\sqrt{f_{n}} \cdot B
$$

(where $f_{n}$ is a constant factor) ensure the equality of $\lambda_{D}^{\prime}=\lambda_{D}$ and $\rho_{e, i}^{\prime}=\rho_{e, i}$. Thus, the the potential and the electric field are related to each other according to

$$
\phi^{\prime}=f_{n} \cdot \phi, \quad E^{\prime}=f_{n} \cdot E
$$

Note, that it is not sufficient to satisfy only $\xi_{e, i}^{\prime}=\xi_{e, i}$. 


\section{Appendix: Dielectric properties}

Using a PIC-model (Sec. (2)) the plasma is treated as a collection of charged particles moving about in their own internal fields. In another approach a plasma can be described as a dielectric medium by considering only gross macroscopic properties (such as the dielectric constant) and not the particle motions themselves.

\section{C.1 The 'plasma capacitor'}

The two different treatments can be readily demonstrated by calculating the energy stored in a simple capacitor. This quantity can be presented as

$$
W=\epsilon W_{o}=\frac{1}{2} \frac{\epsilon \epsilon_{o} S}{d} U^{2}
$$

by using the dielectric constant $\epsilon$ characterizing the dielectric behavior of the used filling. $V=S d$ ist the volume enclosed by the capacitor plates with the area $S$ and the distance between them $d$. $U$ is the applied voltage difference and $W_{o}$ denotes the stored energy for the 'empty' capacitor. In this sence, the dielectric constant $\epsilon$ is defined as the ratio of the electrostatic energy stored per unit volume for a capacitor with material to the same capacitor with only a vacuum as a dielectric.

On the other hand, the stored energy of a capacitor is given in general by

$$
W=\frac{1}{2} \epsilon_{o} \int_{V} E^{2} d V
$$

but the use of Eq. (45) requires the knowledge of the electric field distribution $E(x)$ between the two capacitor plates. It is obtained in a simulation by using the same geometry as described in Sec. A, but instead of the zero boundary condition for the potential at the borders now a voltage $U$ is applied which leads to $\phi(x=0)=-U / 2$ and $\phi(x=L)=+U / 2$. Further, the capacitor is filled with the 'material' plasma extended over the region $L / 4<x \leq 3 L / 4$ between the plates, so that the plasma is kept away from the boundaries in order to avoid the complex interaction at the plasma-electrode interface.

The initital density distributions of particles $e$ and $i$, which are chosen to have charges of opposite sign but the same masses and temperatures, are shown in Fig.18 together with the corresponding distributions of the electric field and potential. The assumed equalities of $m_{e}=m_{i}$ and $T_{e}=T_{i}$ ensure the equality of the gyrations radii $\rho_{e}=\rho_{i}$ preventing, therefore, the occurence of the 'natural' electric field (see Sec. (3)). As expected, the electric field between the plates is constant $|E|=U / d=120 \mathrm{~V} / 0.04 \mathrm{~m}=3000 \mathrm{~V} / \mathrm{m}$ (Fig.18) and $W=W_{o}$. 


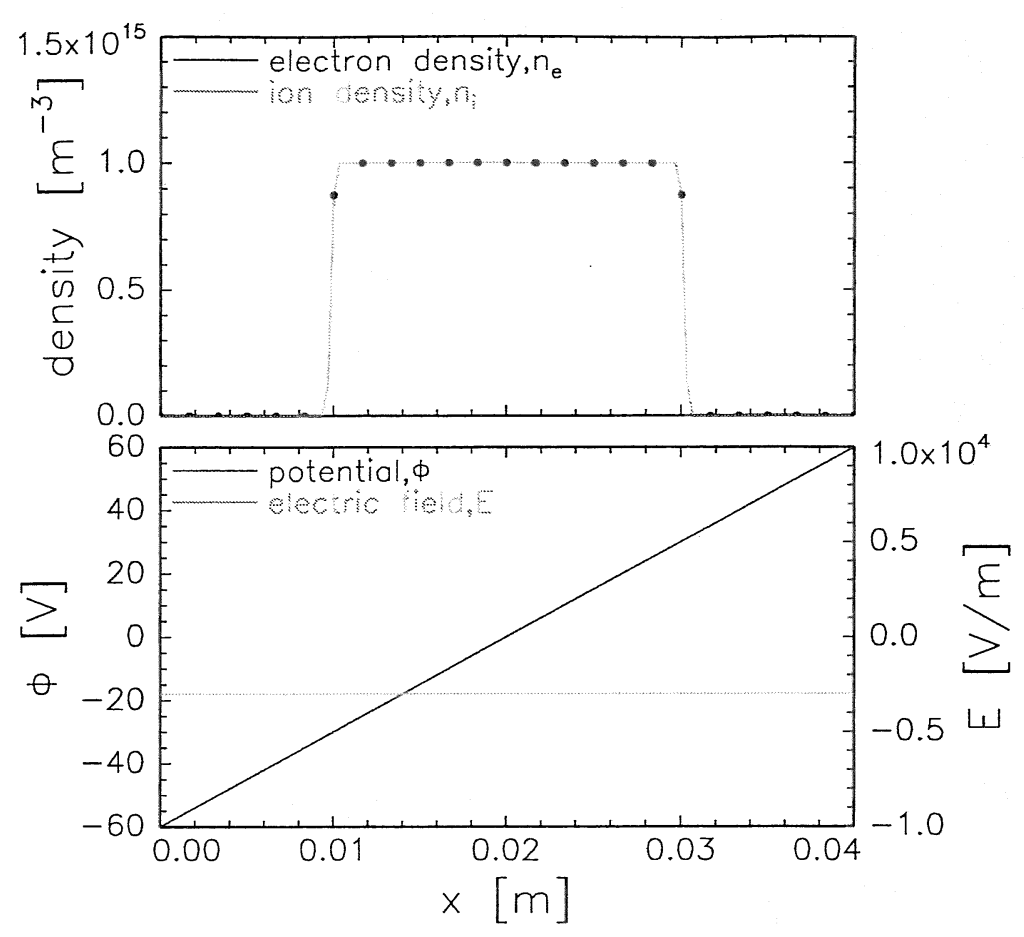

Figure 18: Initial distributions of $n_{i}, n_{e}, \phi$ and $E$ between the two plates of the capacitor. (parameters: $U=120 \mathrm{~V}, d=0.04 \mathrm{~m}, n_{p l}=10^{15} \mathrm{~m}^{-3}, B=0.2 \mathrm{~T}, T_{e}=T_{i}=10 \mathrm{eV}$, $m_{i}=m_{e}=1 \mathrm{amu}, \xi_{i}=\xi_{e}=2, \rho_{i}=\rho_{e}=1.6 \mathrm{~mm}$ )

In the simulation the motion of the particles is then followed in time and steady-state profiles are established after some gyration times. As seen in Fig.19 the density distributions of the charged particles are smoothed at the plasma boundary due to the gyration and are shifted towards the plates, i.e. the particles $i$ with $q=+e$ are attracted towards the negatively charged plate $(x=0)$ and the particles $e$ with $q=-e$ towards the other plate $(\mathrm{x}=\mathrm{L})$. The occurrence of this charge separation leads to a substantial distortion of the potential distribution (Fig.19), the electric field is no longer constant and the integration according to Eq.(45) gives $W=1.8 W_{o}$. So one can conclude, that the dielectric constant of the 'plasma filling' under the analyzed consitions is equal to $\epsilon=1.8$. By varying the magnetic field the main parameter in this analysis $\xi \propto 1 / B$ was changed. The results of these calculations are listed in Table.2. As seen, for strong magnetic fields $\epsilon \rightarrow 1$, i.e. a highly magnetized plasma does not act as a dielectric.

Table 2: Dielectric constant $\epsilon$ as a function of $\xi=\xi_{i}=\xi_{e}$.

\begin{tabular}{|c|c|c|c|c|c|}
\hline$\xi$ & 0.25 & 0.5 & 1 & 1.5 & 2 \\
\hline \hline$\epsilon$ & 1.01 & 1.05 & 1.24 & 1.48 & 1.8 \\
\hline
\end{tabular}

It is important not to mix the two different aproaches, since as long as the motion of particles in their own fields is considered only $\epsilon_{o}$ appears in the Poisson equation (5) and not $\epsilon \epsilon_{o}$. 


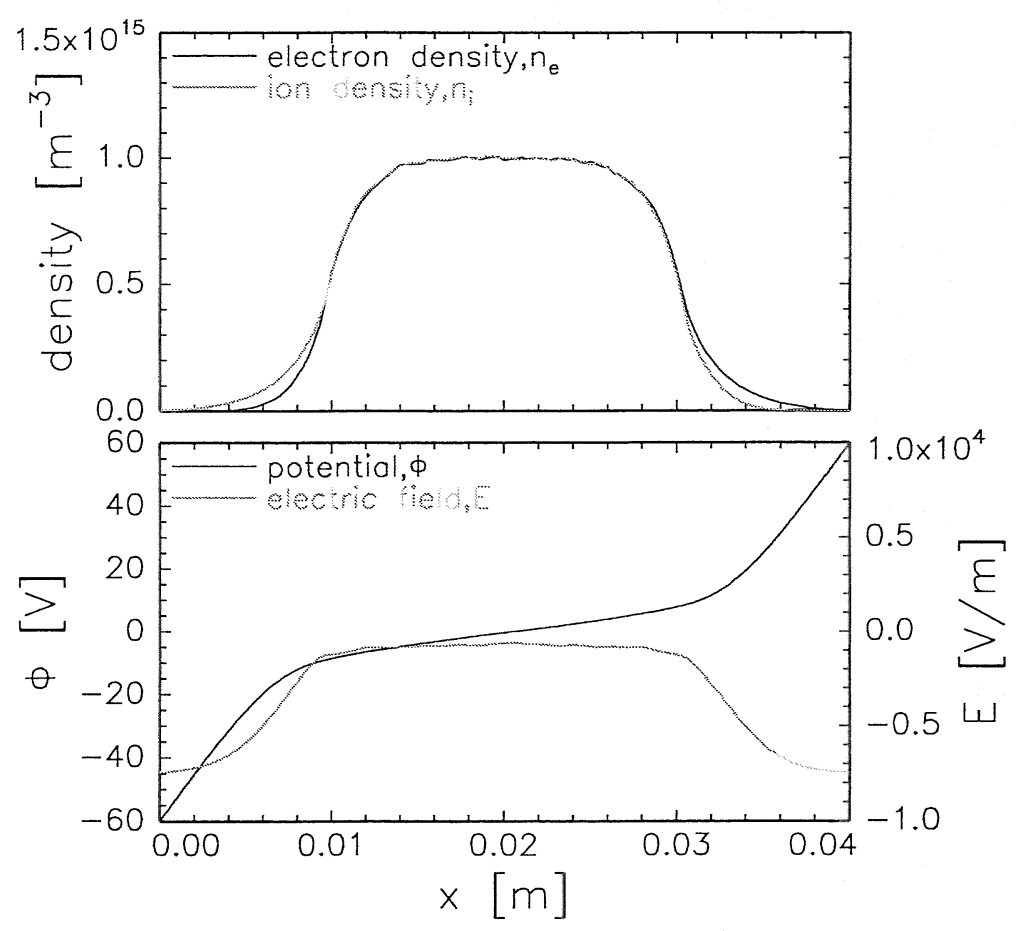

Figure 19: Steady-state distributions of $n_{i}, n_{e}, \phi$ and $E$. (parameters as given in Fig.18)

\section{C.2 Time-varying electric field}

Usually, the dielectric constant of a magnetized plasma is deduced by considering the plasma reaction on a time-varying electric field. The effect of a slowly varying electric field is the addition of the so called polarization drift velocity [8]

$$
v_{p o l}=\frac{m}{q B^{2}} \frac{\partial E}{\partial t}
$$

where $E$ and $v_{p o l}$ are perpendicular to the magnetic field $B$, for example $E=E_{x}, v_{p o l}=$ $v_{p o l, x}$ and $B=B_{z} . v_{p o l}$ is in opposite direction for charges of opposite sign so that the polarization current density in the $x$-direction is given by

$$
j_{p o l, x}=\frac{\left(n_{e} m_{e}+n_{i} m_{i}\right)}{B_{z}^{2}} \frac{\partial E_{x}}{\partial t}
$$

which is dominated by the ions. This current can now be combined with the term $\epsilon_{o} \partial E / \partial t$ which appears on the right-hand side of Maxwell $\nabla \times B$ equation yielding

$$
\epsilon_{o} \frac{\partial E}{\partial t}+\frac{n_{e} m_{e}+n_{i} m_{i}}{B^{2}} \frac{\partial E}{\partial t}=\epsilon_{o} \epsilon \frac{\partial E}{\partial t}
$$

with the dielectric constant

$$
\epsilon=1+\frac{n_{e} m_{e}+n_{i} m_{i}}{\epsilon_{o} B^{2}}=1+\xi_{i}^{2}+\xi_{e}^{2}=1+\chi_{i}+\chi_{e}
$$


(usually $m_{e} \ll m_{i}$ and $\epsilon \approx 1+\xi_{i}^{2}$ ).

In order to prove this relation several plasma simulations have been performed. For a plasma without density gradients and with periodic boundary conditions the validity of relation (49) could be verified.

But the comparison of (49) with the results listed in Table 2 shows a clear disagreement. This means, that at a plasma boundary with strong density gradients (as used in the calculations of the 'plasma capacitor') the relation (49) cannot be used, since in this case the gyration radii are comparable to the density decay length and the derivation leading to $(46,49)$ is not longer valid. Further, for a correct comparison the capacitor should be entirely filled with plasma.

\section{References}

[1] D. Naujoks, Contrib. Plasma Phys., 41, 375 (2001).

[2] C. K. Birdsal and A.B. Langdon, Plasma Physics via Computer Simulation, McGraw-Hill, New York (1985).

[3] J.P. Boris in Proceedings of the 4th Conf. on Numerical Simulation of Plasmas, Edited by J.P. Boris and R.A. Shanny, Naval Research Laboratory, Washington D.C. (1970) p.3-30.

[4] R.W. Hockney, J.W. Eastwood, Computer Simulation using Particles (Adam Hilger, Bristol, Philadelphia, 1988)

[5] R. Courant, K.O. Friedrichs, H. Lewy, Math. Ann. 100 (1928) 32.

[6] A. Kneschke, Differentialgleichungen und Randwertprobleme, Vol. 2, VEB Verlag Technik, Berlin (1960).

[7] G. Fussmann, unpublished (2000).

[8] J. A. Bittencourt, Fundamentals of Plasma Physics, Pergamon Press, Oxford (1986)

[9] S.I. Braginskii, Rev. Plasma Physics, 1, 183 (1963).

[10] N.A. Krall, A.W. Trivelpiece, Principles of Plasma Physics, McGraw-Hill Book Company, New York (1974). 